\title{
Improved Neural Network Adaptive Control for Compound Helicopter with Uncertain Cross- Coupling in Multimodal Maneuver
}

\section{Fengying Zheng}

Nanjing University of Aeronautics and Astronautics

Bowei Xiong ( $D$ 732478220@qq.com )

Nanjing University of Aeronautics and Astronautics https://orcid.org/0000-0002-7206-8608

Jingyang Zhang

Nanjing University of Aeronautics and Astronautics

Ziyang Zhen

Nanjing University of Aeronautics and Astronautics

\section{Feng Wang}

Nanjing University of Aeronautics and Astronautics

\section{Research Article}

Keywords: Compound helicopter, Pi-Sigma neural network, Adaptive control, Incremental dynamic inversion

Posted Date: July 23rd, 2021

DOI: https://doi.org/10.21203/rs.3.rs-714233/v1

License: (c) (1) This work is licensed under a Creative Commons Attribution 4.0 International License. Read Full License

Version of Record: A version of this preprint was published at Nonlinear Dynamics on April 8th, 2022. See the published version at https://doi.org/10.1007/s11071-022-07382-x. 


\title{
Improved neural network adaptive control for compound helicopter with uncertain cross-coupling in multimodal maneuver
}

\author{
Fengying Zheng · Bowei Xiong · Jingyang Zhang · Ziyang Zhen · Feng Wang
}

Received: date / Accepted: date

\begin{abstract}
The main goal of this study was to create a robust control system that could guide or replace the pilots in tracking of commanded velocity and attitude in multimodal maneuver, while complex dynamics and uncertain aerodynamic cross-coupling among control surfaces of compound helicopter are considered. To this end, a Pi-Sigma neural network (PSNN) adaptive controller is proposed based upon the certainty-equivalence (CE) principle, where a novel Lyapunov-based weight self-tuning algorithm augmented with emodifi-cation is designed to realize efficient uncertainty approximation and guarantee robustness of convergence process. Compared with traditional neural networks in control field, stronger generalization ability of PSNN must be balanced against weaker stability, which leads to inevitable parameters perturbation. Therefore, an incremental nonlinear dynamic inversion (INDI) framework is established to decouple original overactuated system and reject parameters perturbation in PSNN. Meanwhile, by incorporating Lagrange multiplier method into allocation, an original incremental allocation method is designed to get globally ideal control input according to time-varying working capability of each surface. In terms of Lyapunov theorem, it is demonstrated that the closed-loop augmented system driven by the proposed control scheme is semi-global uniformly ultimately bounded (SGUUB). Finally, the simulation result validates the effectiveness of proposed control scheme.
\end{abstract}

Keywords Compound helicopter · Pi-Sigma neural network · Adaptive control · Incremental dynamic inversion

Fengying Zheng · Bowei Xiong · Jingyang Zhang $(\bowtie) \cdot$ Feng Wang College of Astronautics, Nanjing University of Aeronautics and Astronautics, Nanjing 210016, China

E-mail: zjy@ nuaa.edu.cn

Ziyang Zhen

College of Automation Engineering, Nanjing University of Aeronautics and Astronautics, Nanjing 210016, China

E-mail: zhenziyang@nuaa.edu.cn

\section{Introduction}

Compound helicopter is a kind of rotorcraft designed to break through maximum speed limit of traditional helicopters (approximately 160-170 knots) and retain capability of vertical take-off/landing. Different from conventional helicopters, the auxiliary propulsion system and wings can help main rotor from dynamic stall on the relating side in high-speed mode. During the past decade, compound helicopters have grown dramatically in both military and civilian fields. However, due to unique characteristics of high speed rotor dynamic, these benefits must be balanced against many disadvantages including time-varying dynamics, mechanical difficulty, unknown aerodynamic interference of additional components, control difficulty and so on $[1,2]$.

In particular, complex aerodynamic cross-coupling among redundancy control surfaces may severely degrade the performance and the stability of compound helicopters. Thus, it is critical to characterize the effect carefully and to alleviate them for reliable autonomous command tracking in multimodal maneuver [3]. Besides, with the increasing complexity of the flight environment, multisource disturbances such as the external disturbances, model uncertainties and unmodeled dynamics also seriously limit the tracking precision of compound helicopters $[4,5]$. Due to factors mentioned above, most missions of compound helicopters can be hardly achieved by traditional controller and design of multimodal command tracking controller becomes a core technique of compound helicopters [6].

Recently, many literatures revealed complex aerodynamic characteristics of compound helicopters and made it possible to build an accurate compound helicopter nominal model which is helpful for control system design [7-10]. However, there are still few related research results to deal with the challenges in advanced controller design. These related literatures mainly consider one certain flight mode such as high- 
speed mode [12] or low-speed mode [13] with traditional model-based approaches [11] including nonlinear dynamic inversion (NDI), sliding mode control (SMC) and backstepping. However, these methods ignored nonaffine property of compound helicopter and can hardly overcome uncertain cross-coupling perturbation throughout flight envelope.

In recent years, there have been various excellent approaches for reference to solve similar problems in command tracking control of complex multi-mode aircraft, such as tilt rotorcraft [14-17], near-space vehicles [18], hypersonic vehicles [19-21] and so on. Within these advanced control methods, neural network (NN) adaptive control is an effective way to handle complex disturbance and model uncertainty in multimodal vehicles [22-25]. However, some factors limit the extension of traditional $\mathrm{NN}$ adaptive control. On one hand, although universal approximation theorem of simple NN has been proved in [26,27], existing NN in control field including sinle hiden layer perceptron (SHL) and radial basis function network (RBFNN) are too simple to efficiently handle uncertain nonlinear function online in complex systems [28]. Furthermore, introduction of excessive neurons in these NN leads to severe co-adaptation and overfitting. On the other hand, some powerful NN such as cerebellar model articulation controller (CMAC) and ridge polynomial neural network (RPNN) usually have heavy structures, which bring too many hyperparameters to design and make these NN much unreliable in engineering [29]. Different from above NN, PSNN is a kind of high order neural network and has received considerable attention recently $[30,31]$ due to its ability of realizing faster nonlinear approximation by introducing both sum and multiplication neurons $[32,33]$. Meanwhile, simple structure and few hyperparameters are needed in PSNN to improve convergence efficiency compared with other complex neural networks [34]. Nowadays PSNN has been applied in various fields [35] and is more suitable for solving control problem in compound helicopter.

In spite of excellent performance of PSNN, research of PSNN adaptive control is still in its infancy. Within these related literatures, PSNN adaptive fuzzy controller proposed in $[36,37]$ is most popular to deal with tracking problem. An offline training process is needed in these fuzzy controllers to approach the optimal membership function. However, such offline training can't adapt to complex environment and unknown interference unavoidable in aerospace engineering. Other literatures mainly focuse on PSNN backstepping approach for a relatively simple system like hydraulic control system [38] to improve control accuracy. Although these methods have been proofed stability, the simple gradient descent adaptive law used in these literatures cannot guarantee the robustness of convergence in more complex systems. The improved PSNN adaptive control strategy for the compound helicopter should consider the following problems: (1) Stability should be ensured strictly in adaptive algorithm since multiplication neurons seriously damage robustness of PSNN; (2) The adaptive PSNN should be augmented with a robust controller architecture to keep robustness to inevitable parameters perturbation in converge process; (3) Practical control input to redundant control surfaces of compound helicopters should be allocated carefully to obtain a efficient tracking performance.

This paper proposes a novel adaptive control scheme for compound helicopters in multimodal maneuver, where aerodynamic cross-coupling among each control surface is considered as partly uncertain disturbances. More specifically, we firstly formulate nonlinear dynamics of a typical compound helicopter and reveal the effect of cross-coupling on control strategy by trim analysis throughout flight envelope. Then, an INDI framework composed of inner attitude control loop and outer velocity control loop is proposed to decouple original overactuated system and keep robustness to inevitable weight perturbation of PSNN. Considering uncertain coupling disturbances, a PSNN adaptive controller agumented with a novel self-tuning adaptive algorithm based on CE principle and Lyapunov theory is proposed approximate undesirable uncertainties. To ensure effiency and robstness, the weight updating law combined with e-modification can improve convergence performance and correct the potential parameter drift in absence of PE. In particular, an original incremental allocation method is determined based on Lagrange multiplier optimization to develop globally optimal input for each control surface based on its time-varying working capability in multimodal maneuver according to a designed allocation matrix satisfying various tasks. It is shown that the tracking errors and weight parameters are SGUUB in terms of Lyapunov theorem. Finally, this study presents a complete Lyapunov stability proof and verifys the efficacy of the proposed approach by numerical simulations.

As compared with previous work, the main contributions of this work can be summarized as follows:

(1) Instead of traditional simple NN adaptive control, PSNN is utilized to uncertain coupling in compound helicopters. To keep robustness to partly uncertain cross-coupling and model error, this study proposes a PSNN Lyapunovbased adaptive algorithm based upon CE principle. Robust adaptive term and e-modification are introduced to correct potential parameters drift and guarantee robustness of convergence process especially in absence of PE. This design can better trade off stablility and nonlinear mapping capability of PSNN compared to existing adaptive law.

(2) Stronger generalization ability of PSNN must be balanced with weaker stability. To address this, an INDIPSNN controller architecture is designed to decouple original overactuated system and minimize effects caused by uncertainties. This controller architecture can signif- 


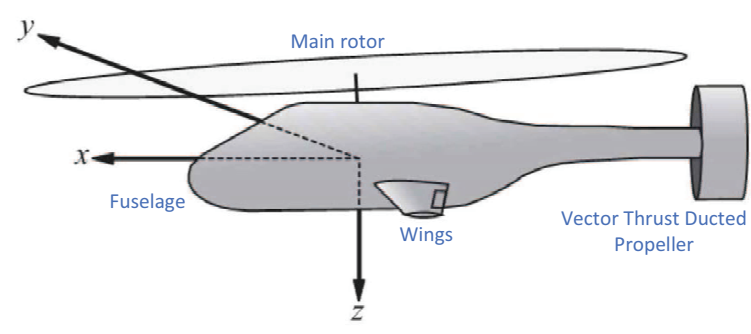

Fig. 1 Structure of a typical compound helicopter

icantly enhance robustness to weight parameters perturbation without requirement of precise mathematical model of compound helicopters.

(3) An incremental Lagrange optimal allocation method is designed to solve control allocation problem considering different working capability of each control surface throughout flight envelope. By extending traditional optimal control allocation methods, this approach optimizes global control efficiency by regulating incremental control input. By selecting appropriate allocation matrix, the ideal control of various task requirements can be achieved.

The remainder of this paper is organized as follows. In Sect 2, we briefly describe compound helicopter nonlinear dynamic model and analyze cross-coupling between control surfaces. In Sect 3, we explain the details of the improved PSNN adaptive control strategy in INDI framework and proof of stability. In Sect 4, we present comparative numerical simulation results with respect to different control strategies. In Sect 5, we conclude the whole paper.

\section{Model statement and Preliminaries}

Notation: $\mathbb{R}^{n}$ is the $\mathrm{n}$-dimensional Euclidean space. $(\cdot)^{\mathrm{T}}$ represents matrix transpose. $\|\cdot\|$ stands for the 2-norm of a vector or Frobenius norm of a matrix. $\sup (\cdot)$ denotes the supremum of $(\cdot) \cdot(\cdot)^{-1}$ stands for the inverse matrix for a nonsingular matrix or one of the pseudo inverse matrices for a singular matrix. Supposing $x \in \mathbb{R}^{n}$ and $f(x) \in \mathbb{R}^{m}, \nabla_{x} f\left(x_{0}\right) \in$ $\mathbb{R}^{m \times n}$ represents the gradient of $f(x)$ to $\mathrm{x}$ at the point $x_{0}$.

In this section, a typical compound helicopter nonlinear dynamic system is first briefly presented with undisirable cross-coupling considered. Thereafter, a trim analysis adjusting to full envelope is given in order to further design the control strategy.

For convenience, choosing the vectored thrust ducted propeller (VTDP) as an auxiliary propulsion device, the main structure of a typical compound helicopter is shown in Fig.1.

Different from conventional helicopters, the compound helicopter is under the action of various forces involving main rotor force $F_{R}$ (mainly acting on low-speed mode), VTDP force $F_{T}$, fuselage force $F_{f}$, wing force $F_{w}$ (mainly acting on high-speed mode) and gravity $G$. Based on the momentum theorem and the moment of momentum theorem, the compound helicopter dynamic model can be obtained as

$m\left[\begin{array}{c}\dot{u}+q w-v r \\ \dot{v}+u r-p w \\ \dot{w}+p v-u q\end{array}\right]=F_{R}+F_{T}+F_{f}+F_{w}+G$

$\left[\begin{array}{l}J_{x} \dot{p}+q r\left(J_{z}-J_{y}\right) \\ J_{y} \dot{q}+\operatorname{pr}\left(J_{x}-J_{z}\right) \\ J_{z} \dot{r}+p q\left(J_{y}-J_{x}\right)\end{array}\right]=M_{R}+M_{T}+M_{f}+M_{w}$

where $(u, v, w)$ and $(p, q, r)$ are velocity components and angular velocity components in body-fixed frame respectively; $\left(J_{x}, J_{y}, J_{z}\right)$ are inertia moment in body-fixed frame. $M_{R}, M_{T}$, $M_{f}$ and $M_{w}$ are the corresponding torques. In this study, to analyze control characteristics of compound helicopter, nonlinear dynamics as well as coupling effects of main rotor, wings and VTDP will be briefly introduced. Complete dynamic model is introduced in [39].

\subsection{Main rotor nonlinear dynamic}

Different from linear assumption in other literatures, flapping motion of main rotor across flight envelope is described by a second order differential equation as

$\ddot{\beta}_{i}=\frac{M_{b}}{I_{b}} \sin \beta_{i}\left(-e \Omega^{2}\right)-\cos \beta_{i} \sin \beta_{i} \Omega^{2}+\frac{\left(M_{\mathrm{FAB}}\right)_{i}-k_{\beta} \beta_{i}}{I_{b}}$

where the subscript $i$ represents the parameters on the ith blade, $\beta$ denotes blade flapping angle, $M_{b}$ is aerodynamic torque acting on blade, $I_{b}$ is blade inertia, $e$ is flapping hinge offset, $\Omega$ is main rotor speed, $M_{\mathrm{FAB}}$ is the sum of the aerodynamic torque acting on the flapping hinge and $k_{\beta}$ is flapping coefficient. Then, according to the blade-element theory, aerodynamic force and torque expressions of main rotor are calculated as

$$
\begin{aligned}
& \left\{\begin{aligned}
\left(F_{P b}\right)_{i}= & \sum_{k=1}^{N S}\left[\frac{1}{2} \rho \Omega^{2} R^{3} U\left(C_{y} \frac{U_{T}}{|\cos \gamma|}+C_{d} U_{P}\right) \mathrm{d} s\right]_{k} \\
\left(F_{T b}\right)_{i}= & \sum_{k=1}^{N S}\left[\frac{1}{2} \rho \Omega^{2} R^{3} U\left(C_{d} U_{T}-C_{y} U_{P}|\cos \gamma|\right) \mathrm{d} s\right]_{k} \\
\left(F_{R b}\right)_{i}= & \sum_{k=1}^{N S}\left[\frac{1}{2} \rho \Omega^{2} R^{3} U\left(C_{d}-C_{y} \frac{U_{P}|\cos \gamma|}{U_{T}}\right) \mathrm{d} s\right]_{k}
\end{aligned}\right. \\
& \left\{\begin{aligned}
\left(F_{X}\right)_{i}= & -\left(F_{T b}\right)_{i}+2 M_{b} \Omega \dot{\beta}_{i} \sin \beta_{i} \\
\left(F_{Y}\right)_{i}= & \left(F_{R b} \cos \beta-F_{P b} \cos \beta\right)_{i}+M_{b}\left[\cos \beta_{i}\left(\dot{\beta}_{i}^{2}+\Omega^{2}\right)\right. \\
& \left.+\operatorname{sim} \beta_{i} \ddot{\beta}_{i}\right] \\
\left(F_{Z}\right)_{i}= & \left(F_{P b} \sin \beta-F_{P b} \cos \beta\right)_{i}+M_{b}\left[\ddot{\beta}_{i} \cos \beta_{i}-\ddot{\beta}_{i} \cos \beta_{i}\right. \\
& \left.-\dot{\beta}_{i}^{2} \sin \beta_{i}\right]
\end{aligned}\right.
\end{aligned}
$$


where $F_{P b}, F_{T b}$ and $F_{R b}$ are blade aerodynamic forces in vertical, tangential and spanwise directions, respectively; $F_{X}$, $F_{Y}$ and $F_{Z}$ are load components of blade in rotation axis; $N S$ is number of elements on blade; $\rho$ is air density; $R$ is blade elements rotation radius; $U_{T}, U_{P}$ and $U_{R}$ are tangential, vertical and spanwise velocity components at an element of the blade; $U=\left(U_{T}^{2}+U_{P}^{2}+U_{R}^{2}\right)^{1 / 2}$ is total velocity of blade; $\gamma$ is blade profile drift angle; $C_{y}$ and $C_{d}$ are lift coefficient and drag coefficient got from wind tunnel test data; $\mathrm{d} s$ is the area of each blade element.

To sum up, the force and torque generated by the main rotor throughout flight envelope can be written as

$$
\begin{gathered}
F_{R}=\left[\begin{array}{c}
-\sum_{i=1}^{N}\left(F_{z}\right)_{i} \\
\sum_{i=1}^{N}\left(F_{Y} \cos \varphi-F_{X} \sin \varphi\right)_{i} \\
-\sum_{i=1}^{N}\left(F_{X} \cos \varphi+F_{Y} \sin \varphi\right)_{i}
\end{array}\right] \\
M_{R}=\left[\begin{array}{c}
\sum_{i=1}^{N} e\left(F_{Z} \cos \varphi\right)_{i} \\
\sum_{i=1}^{N} e\left(F_{Z} \sin \varphi\right)_{i} \\
-\sum_{i=1}^{N}\left(e F_{X}-M_{\mathrm{LAB}} \cos \beta\right)_{i}
\end{array}\right]
\end{gathered}
$$

where $M_{\mathrm{LAB}}$ is the sum of the aerodynamic moment acting on the shimmy hinge, $\varphi$ is the rotor azimuth and $N$ is the number of blades.

\subsection{Wings nonlinear dynamic}

Supposing that the right-wing center is $\left(x_{w r}, y_{w r}, z_{w r}\right)$ in bodyfixed frame, the velocity of right-wing can be described as

$V_{w r}=\left[\begin{array}{c}u+q z_{w r}-r y_{w r}+v_{i} \zeta_{x_{\mathrm{MRW}}} \\ v-p z_{w r}-r x_{w r} \\ w+p y_{w r}-q x_{w r}+v_{i} \zeta_{z_{\mathrm{MRW}}}\end{array}\right]$

where $v_{i}$ is rotor inducted velocity, $\zeta_{x_{\mathrm{MRW}}}$ and $\zeta_{z_{\mathrm{MRW}}}$ are the cross-coupling factors of rotor downwash shown in Fig.2 and Fig.3, where $\beta_{1 s}$ is rotor flapping skew angle.

The aerodynamic force and torque of the wind frame can be expressed as

$$
\begin{aligned}
& {\left[\begin{array}{l}
F_{w r}^{x} \\
F_{w r}^{y} \\
F_{w r}^{z}
\end{array}\right]=L_{B}^{W}\left(V_{w r}\right)\left[\begin{array}{c}
-C_{D w r}\left(\alpha_{w r}, \delta_{r}\right) q_{w r} S \\
0 \\
-C_{L w r}\left(\alpha_{w r}, \delta_{r}\right) q_{w r} S
\end{array}\right]} \\
& {\left[\begin{array}{c}
M_{w r}^{x} \\
M_{w r}^{y} \\
M_{w r}^{z}
\end{array}\right]=L_{B}^{W}\left(V_{w r}\right)\left[\begin{array}{c}
0 \\
C_{M w r}\left(\alpha_{w r}, \delta_{r}\right) q_{w r} S b \\
0
\end{array}\right]}
\end{aligned}
$$

where $L_{B}^{W}\left(V_{w r}\right)$ is the coordinate transformation matrix of wind frame in body frame; $C_{D w r}, C_{L w r}, C_{M w r}$ are wings coefficient functions of drag, lift and yawing torque; $S$ is stabilizing surface area; $b$ is wing span; $\alpha_{w r}$ is attack angle of right-wing; $q_{w r}$ is dynamic pressure of right-wing.

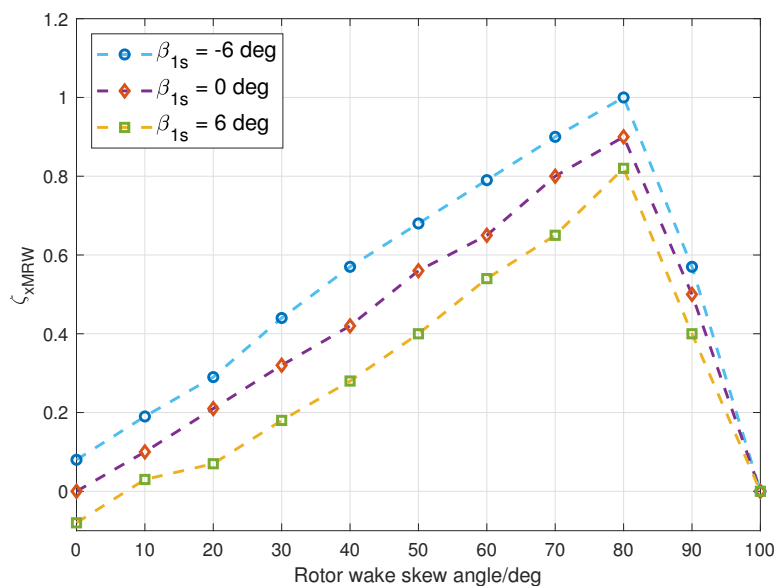

Fig. 2 Cross-coupling factor of rotor to wings in $x$ axis

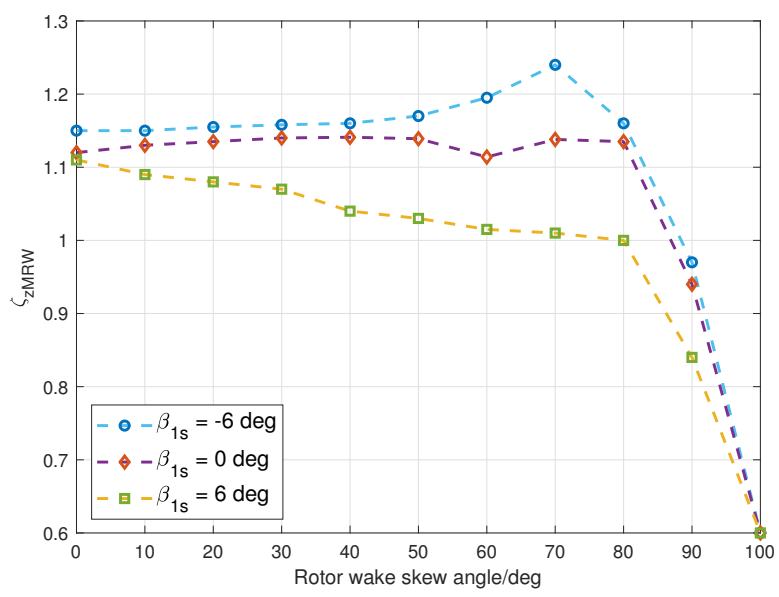

Fig. 3 Cross-coupling factor of rotor to wings in $z$ axis

In the same way, aerodynamic force and torque of leftwing $F_{w l}^{x}, F_{w l}^{y}, F_{w l}^{z}, M_{w l}^{x}, M_{w l}^{y}, M_{w l}^{z}$ can be obtained, and the dynamic of wings can be described as

$$
\begin{aligned}
F_{w}= & {\left[\begin{array}{l}
F_{w r}^{x} \\
F_{w r}^{y} \\
F_{w r}^{z}
\end{array}\right]+\left[\begin{array}{c}
F_{w l}^{x} \\
F_{w l}^{y} \\
F_{w l}^{z}
\end{array}\right] } \\
M_{w}= & {\left[\begin{array}{c}
M_{w r}^{x} \\
M_{w r}^{y} \\
M_{w r}^{z}
\end{array}\right]+\left[\begin{array}{c}
M_{w l}^{x} \\
M_{w l}^{y} \\
M_{w l}^{z}
\end{array}\right]+\left[\begin{array}{c}
-F_{w r}^{y} z_{w r}+F_{w r}^{z} y_{w r} \\
-F_{w r}^{z} x_{w r}+F_{w r}^{x} z_{w r} \\
F_{w r}^{y} z_{w r}
\end{array}\right] } \\
+ & {\left[\begin{array}{c}
-F_{w w}^{y} z_{w l}+F_{w l}^{z} y_{w l} \\
-F_{w l}^{z} x_{w l}+F_{w l}^{x} z_{w l} \\
F_{w l}^{y} z_{w l}
\end{array}\right] }
\end{aligned}
$$

\subsection{VTDP nonlinear dynamic}

The VTDP dynamic in body axis can be obtained by assuming that VTDP can make small angle variation of two degree of freedom in thrust direction. Let the pressure center of VTDP be $\left(x_{t}, y_{t}, z_{t}\right)$. The approaching velocity in duct 


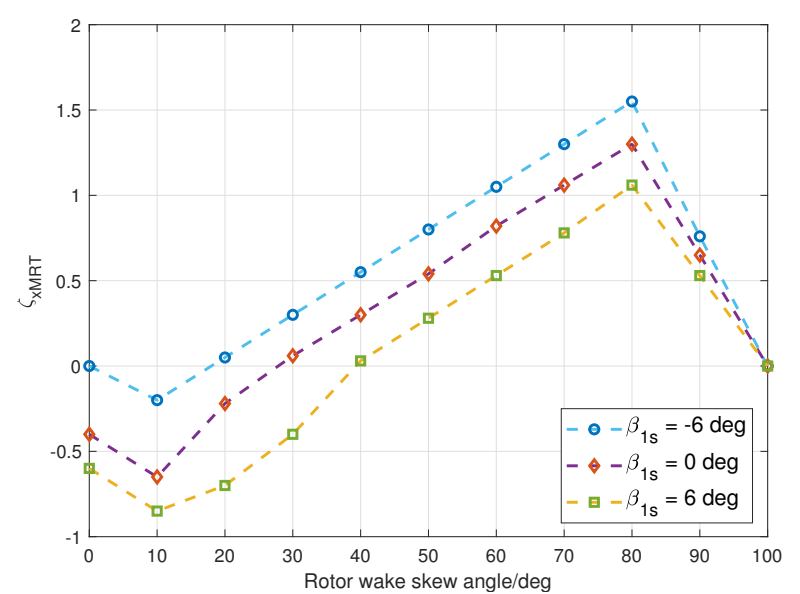

Fig. 4 Cross-coupling factor of rotor to VTDP in $x$ axis

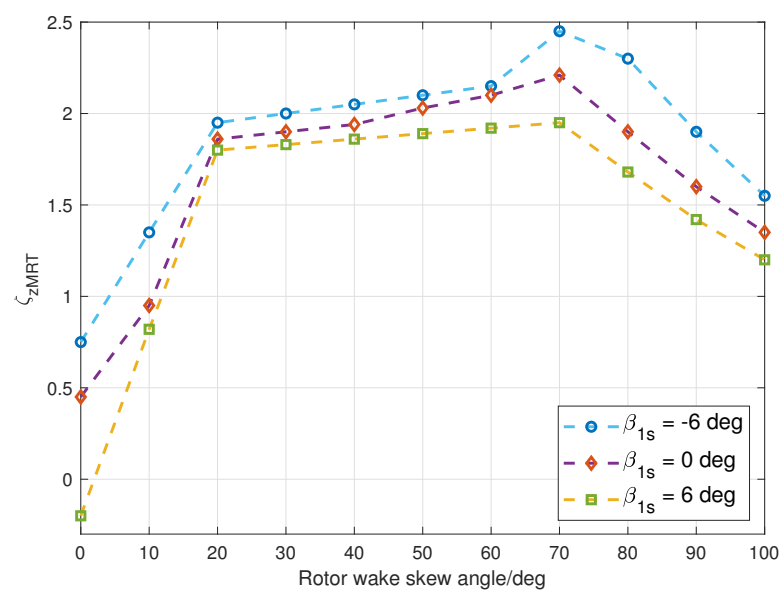

Fig. 5 Cross-coupling factor of rotor to VTDP in $z$ axis

frame is

$V_{T}=\left[\begin{array}{c}k_{q} u+q z_{t}-r y_{t}+v_{i} \zeta_{x_{\mathrm{MRT}}} \\ k_{q} v+p z_{t}-r x_{t} \\ k_{q} w+p y_{t}-q x_{t}+v_{i} \zeta_{z_{\mathrm{MRT}}}\end{array}\right]=\left[\begin{array}{c}V_{x t} \\ V_{y t} \\ V_{z t}\end{array}\right]$

where $k_{q}$ is pressure-lose coefficient of the ducted fan, $\zeta_{x_{\mathrm{MRT}}}$ and $\zeta_{z_{\mathrm{MRT}}}$ are the cross-coupling factors of rotor to VTDP shown in Fig.4 and Fig.5. Transform the components of VTDP thrust in duct frame to body fixed frame, and dynamic of VTDP can be described as

$F_{T}=L_{B}^{T}\left(\theta_{1}, \theta_{2}, V_{T}\right)\left[\begin{array}{c}T \\ \frac{1}{2} \rho\left\|V_{T}\right\|^{2} Q_{s y} \arctan \frac{V_{z t}}{\sqrt{V_{x t}^{2}+V_{y t}^{2}}} \\ \frac{1}{2} \rho\left\|V_{T}\right\|^{2} Q_{s z} \arctan \frac{V_{y t}}{V_{x t}}\end{array}\right]$

$M_{T}=\left[\begin{array}{ccc}0 & -z_{t} & y_{t} \\ z_{t} & 0 & -x_{t} \\ y_{t} & z_{t} & 0\end{array}\right] F_{T}$

where $Q_{s y}$ and $Q_{s z}$ are airflow slip coefficients, the $L_{B}^{T}$ is coordinate transformation matrix controlled by VTDP deflection $\theta_{1}, \theta_{2}$ and $V_{T}$

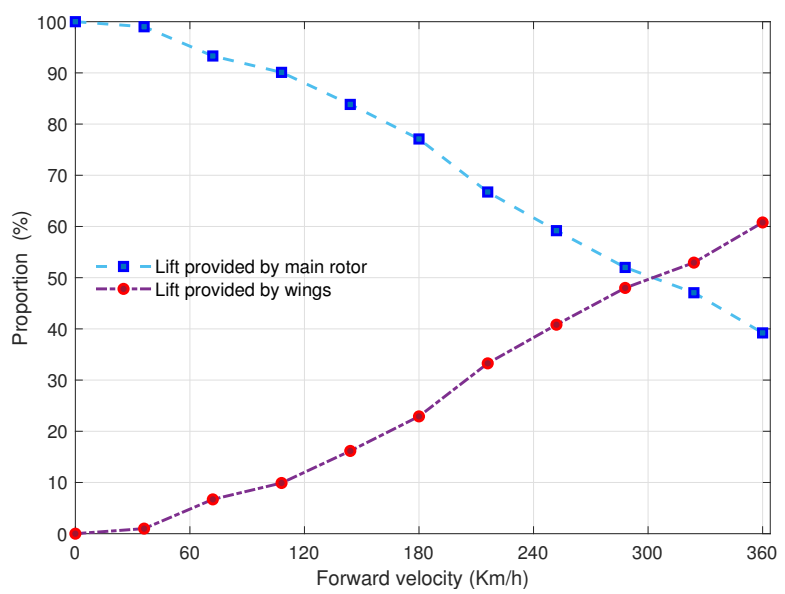

Fig. 6 Variety of lift propotion provided by main rotor and wings in full envelope

\subsection{Trim analysis}

In summary, the compound helicopter dynamic model is obtained as

$$
\begin{aligned}
m\left[\begin{array}{c}
\dot{u} \\
\dot{v} \\
\dot{w}
\end{array}\right] & =\left[\begin{array}{c}
v r-q w-m g \sin \theta \\
p w-u r-m g \cos \theta \sin \phi \\
u q-p v+m g \cos \phi \cos \theta
\end{array}\right]+F_{f} \\
& +F_{R}\left(\varphi_{7}, A_{1 s}, B_{1 s}\right)+F_{T}\left(T, \theta_{1}, \theta_{2}, v_{i}\right) \\
& +F_{w}\left(\delta_{r}, \delta_{l}, v_{i}\right) \\
J\left[\begin{array}{c}
\dot{p} \\
\dot{q} \\
\dot{r}
\end{array}\right]= & {\left[\begin{array}{c}
\left(J_{y}-J_{z}\right) q r \\
\left(J_{z}-J_{x}\right) p r \\
\left(J_{x}-J_{y}\right) p q
\end{array}\right]+M_{f}+M_{R}\left(\varphi_{7}, A_{1 s}, B_{1 s}\right) } \\
+ & M_{T}\left(T, \theta_{1}, \theta_{2}, v_{i}\right)+M_{w}\left(\delta_{r}, \delta_{l}, v_{i}\right)
\end{aligned}
$$

where control surfaces include collective $\varphi_{7}$, lateral cyclic $A_{1 s}$, longitudinal cyclic $B_{1 s}$, deflection angle of VTDP relative to longitudinal symmetry plane $\theta_{1}$, deflection angle of VTDP projection on longitudinal symmetry plane relative to transverse symmetry plane $\theta_{2}$, left-wing deflection angle $\delta_{l}$, right-wing deflection angle $\delta_{r}$ and VTDP thrust $T$. The forces and torques are not only nonlinear functions of control surfaces, but also functions of flight states and the rotor inducted velocity $v_{i}$, which is shown above.

The efficiencies of each control surface vary in multimodal maneuver. When flight speed increases and dynamic pressure $q$ increases, the lift and torques generated by the wing grow in quadratic function. Meanwhile, the aerodynamic characteristics of the rotor gradually show strong nonlinearity, and blade dynamic stall occur due to unsteady flapping motion $\beta$. To be specific, a detailed trim analysis is given in this study.

The trim optimization of flight state from hovering to $360 \mathrm{~km} / \mathrm{h}$ is conducted using Sequential Quadratic Programming (SQP), and optimal variety of control surfaces deflection in different flight mode is obtained. Under constraint of 


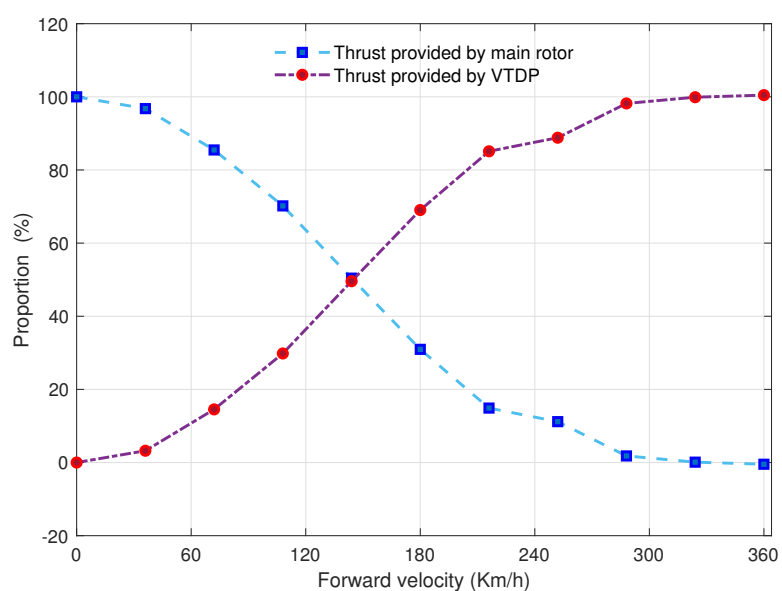

Fig. 7 Variety of thrust propotion provided by main rotor and VTDP in full envelope

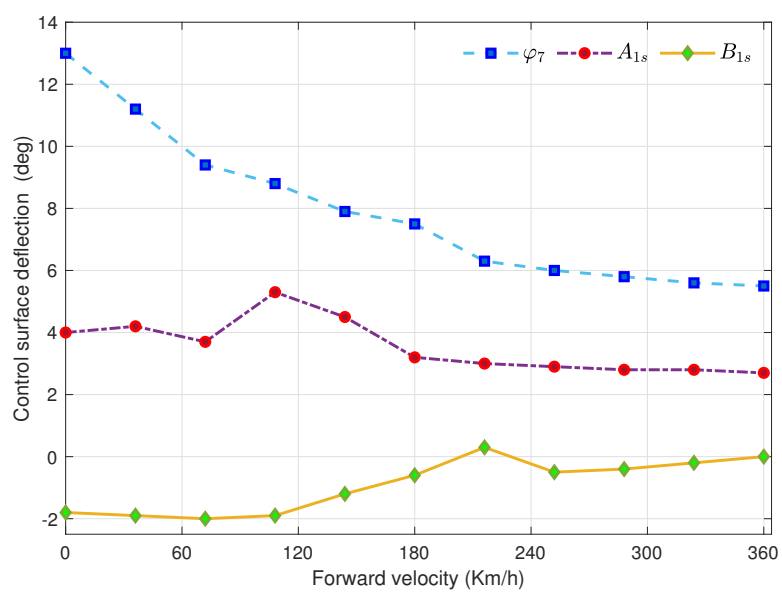

Fig. 8 Variety of $\varphi_{7}, A_{1 s}, B_{1 s}$ in full envelope

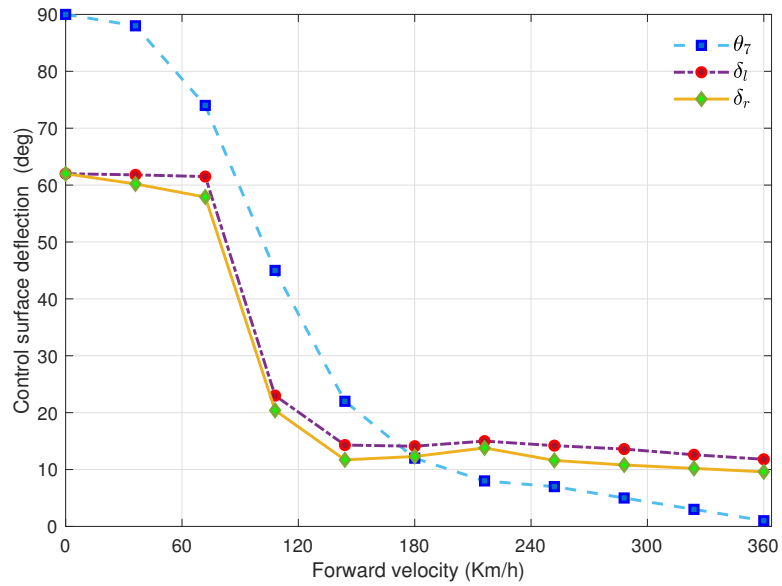

Fig. 9 Variety of $\theta_{1}, \delta_{l}, \delta_{r}$ in full envelope ensuring the balance of external force and torque, the objective function of trim optimization is designed as $J_{\text {trim }}=$ $u^{\mathrm{T}} H u$, where $H$ is optimization matrix. The trim results are given below.

Fig. 6 and Fig. 7 show that the lift and thrust are mainly provided by main rotor in low-speed mode. The loading on the rotor reduces and the wings auxiliary lift as dynamic pressure increases. In high-speed mode, the rotor takes only $40 \%$ of lift, while the wing supplements $60 \%$. Meanwhile, thrust is completely supplemented by VTDP, which embodies the compounding of the lift and thrust. It is worthwhile noting that these variations are nonlinear.

Fig. 8 and Fig. 9 show the trim values of control surfaces in different flight mode. Fig.8 shows that optimal collective, lateral cyclic, and longitudinal cyclic approach zero in highspeed mode to reduce the negative effect of blade stall. Fig.9 shows that the wings control surfaces keep large deflection angle in low-speed flight to reduce wings area and influence of rotor downwash. With the increase of dynamic pressure, they gradually undertake the task of attitude control and approach their normal workspace. Furthermore, in low-speed mode, VTDP deflection maintains large angle to undertake the task of helicopter tail rotor with low thrust. As the airspeed increases, VTDP power also increases and the deflection decreases to provide sufficient thrust.

From the above analysis, it is known that the control efficiency of each control surface has time-varying nonlinear characteristics in multimodal maneuver, and complex crosscouplings between each part also bring lots of challenges to controller design.

\section{Main results}

For convenience, the compound helicopter nonaffine nonlinear dynamic system $(10)(11)$ can be represented as two subsystems

$\dot{x}_{1}=f_{1}\left(x_{1}, x_{2}\right)+g_{1}\left(x_{1}, x_{2}, u_{1}\right)$

$\dot{x}_{2}=f_{2}\left(x_{2}\right)+g_{2}\left(x_{2}, u_{2}\right)$

where $x_{1}=[u, v, w]^{\mathrm{T}}$ are relatively slow state variables, $x_{2}=$ $[p, q, r]^{\mathrm{T}}$ are relatively fast state variables, $u_{1}=\left[\phi_{c m d}, \theta_{c m d}, \varphi_{7}\right.$ $, T]^{\mathrm{T}}$ are control inputs in slow subsystem and $u_{2}=\left[A_{1 s}, B_{1 s}\right.$, $\left.\delta_{l}, \delta_{r}, \theta_{1}, \theta_{2}\right]^{\mathrm{T}}$ are control inputs in fast subsystem. Owing to partly uncertain coupling and variations of aerodynamic models, it's difficult to obtain system parameters by measurement and theoretical calculation. A nominal model is used to approximate the real compound helicopter model

$$
\begin{aligned}
& \dot{x}_{1}=\hat{f}_{1}\left(x_{1}, x_{2}\right)+\hat{g}_{1}\left(x_{1}, x_{2}, u_{1}\right)+\bar{\Delta}_{1}\left(x_{1}, x_{2}, u_{1}\right) \\
& \dot{x}_{2}=\hat{f}_{2}\left(x_{2}\right)+\hat{g}_{2}\left(x_{2}, u_{2}\right)+\bar{\Delta}_{2}\left(x_{2}, u_{2}\right)
\end{aligned}
$$


where the superscript ${ }^{\wedge}$ denotes nominal model and the function $\bar{\Delta}_{i}$ is uncertain model defined as

$\bar{\Delta}_{i}\left(x_{i}, u_{i}\right) \triangleq f_{i}\left(x_{i}\right)+g_{i}\left(x_{i}, u_{i}\right)-\hat{f}_{i}\left(x_{i}\right)-\hat{g}_{i}\left(x_{i}, u_{i}\right)$

Assumption 1 The considered nonlinear function $f_{i}(\cdot)$ satisfies global Lipschitz condition, that is

$$
\begin{aligned}
& \left\|f_{i}\left(x_{1}\left(k_{1}\right), x_{2}\left(k_{1}\right)\right)-f_{i}\left(x_{1}\left(k_{2}\right), x_{2}\left(k_{2}\right)\right)\right\| \\
& \leq L_{1}\left|x_{1}\left(k_{1}\right)-x_{1}\left(k_{2}\right)\right|+L_{2}\left|x_{2}\left(k_{1}\right)-x_{2}\left(k_{2}\right)\right|
\end{aligned}
$$

Similar conclusions apply to $\hat{f}_{i}(\cdot), g_{i}(\cdot), \hat{g}_{i}(\cdot)$.

Assumption 2 The partial derivative of $\hat{g}_{i}(\cdot)$ with respect to control input exits and the sign is unchanged. That is $\partial \hat{g}_{i}(\cdot) / \partial u_{i} \geq l$ (or $\partial \hat{g}_{i}(\cdot) / \partial u_{i} \leq-l$ ), where $u_{i}$ denotes control input, $l>0$ is a small constant. In following discussion, we consider the case of $\partial \hat{g}_{i}(\cdot) / \partial u_{e} \geq l$

In this paper, a nonlinear controller is designed to realize the command tracking control of the compound helicopter in multimodal maneuver. The objective is to find control inputs $u_{1}(t)$ and $u_{2}(t)$, such that $x_{1}(t)$ accurately tracks the desired command $x_{1 \text { cmd }}(t)=\left[u_{c m d}(t), v_{c m d}(t), w_{c m d}(t)\right]^{\mathrm{T}}$ and $\psi_{c m d}$ in multimodal maneuver.

Assumption 3 Let the external commanded input and its first and second derivatives be bounded, such that

$\left\|\left(u_{c m d} \dot{u}_{c m d} \ddot{u}_{c m d}\right)^{\mathrm{T}}\right\| \leq \bar{U}_{C}$

Similar assumptions are made for all channels in $x_{1 \mathrm{cmd}}$ and $\psi_{\text {cmd }}$.

Since the flight state variables can be separated into fast and slow variables in terms of singular perturbed theory, we firstly adopt two control loop based on INDI architecture. Thereafter, an improved PSNN adaptive controller is proposed to realize robust tracking control in multimodal maneuver. Finally, in terms of Lyapunov theorem, it's demonstrated that the close-loop system is GUUB. To summarize the proposed controller, a flow diagram is shown in Fig.10.

\subsection{INDI control framework}

\subsubsection{Outer control loop design}

The objective of the outer-loop velocity controller is to follow the velocity command $x_{1 \mathrm{cmd}}$ and generate desired commands $\theta_{c m d}$ and $\phi_{c m d}$ for the attitude controller. In addition, to follow the velocity command, it generates the collective input $\varphi_{7}$ for the rotor and the desired thrust $T$ for the thrust ducted propeller.

In order to obtain the incremental form of the compound helicopter velocity dynamic model, (12) are rewritten by applying the Taylor series expansion to the $\hat{g}_{i}\left(x_{1}, x_{2}, u_{1}\right)$ at the

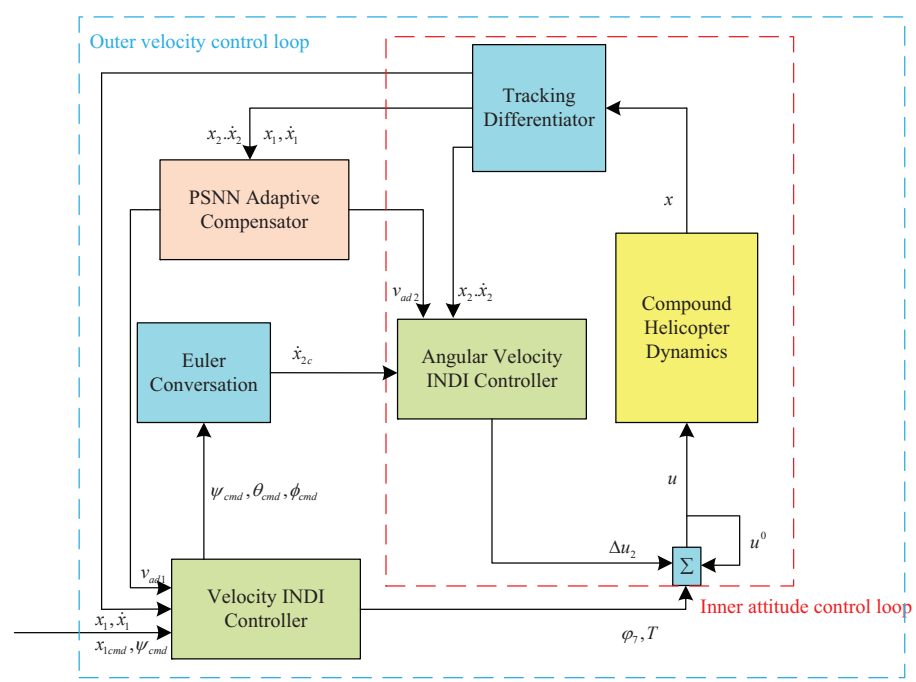

Fig. 10 Overview of proposed control architecture

beginning of each sampling interval (denoted by superscript 0)

$$
\begin{aligned}
\dot{x}_{1}= & \hat{f}_{1}\left(x_{1}, x_{2}\right)+\hat{g}_{1}\left(x_{1}^{0}, x_{2}^{0}, u_{1}^{0}\right) \\
& +\nabla_{\left(x_{1}, x_{2}, u_{1}\right)} \hat{g}_{1}\left(x_{1}^{0}, x_{2}^{0}, u_{1}^{0}\right) \cdot\left(\Delta x_{1}, \Delta x_{2}, \Delta u_{1}\right) \\
& +R_{1}^{\prime}\left(\Delta x_{1}, \Delta x_{2}, \Delta u_{1}\right)+\bar{\Delta}_{1}\left(x_{1}, x_{2}, u_{1}\right)
\end{aligned}
$$

in which the increments of the variables with respect to their current values are denoted by the notation $\Delta, R_{1}^{\prime}\left(\Delta x_{1}, \Delta x_{2}\right.$, $\left.\Delta u_{1}\right)$ is the higher order remainder of Taylor expansion. By introducing acceleration feedback from tracking differentiator, (15) is then written as

$$
\begin{aligned}
\dot{x}_{1}= & \dot{x}_{1}^{0}-\hat{f}_{1}\left(x_{1}^{0}, x_{2}^{0}\right)-\bar{\Delta}_{1}\left(x_{1}^{0}, x_{2}^{0}, u_{1}^{0}\right)+\hat{f}_{1}\left(x_{1}, x_{2}\right) \\
& +\nabla_{\left(x_{1}, x_{2}, u_{1}\right)} \hat{g}_{1}\left(x_{1}^{0}, x_{2}^{0}, u_{1}^{0}\right) \cdot\left(\Delta x_{1}, \Delta x_{2}, \Delta u_{1}\right) \\
& +R^{\prime}{ }_{1}\left(\Delta x_{1}, \Delta x_{2}, \Delta u_{1}\right)+\bar{\Delta}_{1}\left(x_{1}, x_{2}, u_{1}\right)
\end{aligned}
$$

Assumption 4 Without loss of generality, the changes of system states including $x_{1}$ and $x_{2}$ are approximately negligible in a short time increment $T_{s}$. Such that $\lim _{T_{s} \rightarrow 0} x_{i}=x_{i}^{0}$.

Based on this assumption, (16) becomes

$\dot{x}_{1}=\dot{x}_{1}^{0}+\nabla_{u_{1}} \hat{g}_{1}\left(x_{1}, x_{2}, u_{1}\right) \cdot \Delta u_{1}+R_{1}\left(\Delta u_{1}\right)+\Delta_{1}\left(\Delta u_{1}\right)$

where $\Delta_{1}$ is defined as incremental model error depending on $\Delta u_{1}$ and $R_{1}$ is defined as incremental higher order perturbing term mainly depending on high order term of $\Delta u_{1}$. Thus, the INDI control law of velocity loop is designed by using the NDI based on (17) in every sampling interval given by

$$
\begin{aligned}
& \Delta u_{1}=\left[\nabla_{u_{1}} \hat{g}_{1}\left(x_{1}, x_{2}, u_{1}^{0}\right)\right]^{-1}\left(\dot{x}_{1 c}+v_{\mathrm{L} 1}-\dot{x}_{1}\right) \\
& u_{1}=u_{1}^{0}+\Delta u_{1}
\end{aligned}
$$


where $\dot{x}_{1 c}$ is obtained from reference model designed according to the requirements of performance index, $\left[\nabla_{u_{1}} \hat{g}_{1}\left(x_{1}\right.\right.$ $\left.\left., x_{2}, u_{1}^{0}\right)\right]^{-1}$ denotes one of the pseudo inverse matrices designed below, the linear pseudo control signal $v_{\mathrm{L} 1}$ is designed as follows

$v_{\mathrm{L} 1}=K_{\mathrm{P} 1}\left[\begin{array}{c}u_{c}-u \\ v_{c}-v \\ w_{c}-w\end{array}\right]+K_{\mathrm{I} 1} \int_{t_{0}}^{t}\left[\begin{array}{c}u_{c}-u \\ v_{c}-v \\ w_{c}-w\end{array}\right] \mathrm{d} \tau$

where $K_{\mathrm{P} 1}$ and $K_{\mathrm{I} 1}$ are linear control coefficients.

\subsubsection{Inner control loop design}

To follow Euler angle command $\phi_{c m d}(t), \theta_{c m d}(t)$ and $\psi_{c m d}(t)$ obtained from velocity controller and yaw angle command, the angular velocity command is achieved by Euler conversation $\dot{x}_{2 c m d}=\left(\Omega_{r}\left[\phi_{c m d}(t), \theta_{c m d}(t), \psi_{c m d}(t)\right]^{\mathrm{T}}\right)^{\prime}$, where $\Omega_{r}$ is corresponding coordinate rotation matrix.

Similar to the velocity control, Taylor series expansion is applied again to obtain the incremental form of the compound helicopter velocity dynamic model

$$
\begin{aligned}
\dot{x}_{2}= & \hat{f}_{2}\left(x_{2}\right)+\nabla_{\left(x_{2}, u_{2}\right)} \hat{g}_{2}\left(x_{2}^{0}, u_{2}^{0}\right) \cdot\left(\Delta x_{2}, \Delta u_{2}\right)+\hat{g}_{2}\left(x_{2}^{0}, u_{2}^{0}\right) \\
& +R_{2}^{\prime}\left(\Delta x_{2}, \Delta u_{2}\right)+\bar{\Delta}_{2}\left(x_{2}, u_{2}\right)
\end{aligned}
$$

where $R_{2}^{\prime}\left(\Delta x_{2}, \Delta u_{1}\right)$ is the higher order remainder of Taylor expansion. Through the introduction of angular acceleration feedback, (20) is then written as

$$
\begin{aligned}
\dot{x}_{2}= & \dot{x}_{2}^{0}-\hat{f}_{2}\left(x_{2}^{0}\right)-\bar{\Delta}_{2}\left(x_{2}^{0}, u_{2}^{0}\right)+\hat{f}_{2}\left(x_{2}\right)+R_{2}^{\prime}\left(\Delta x_{2}, \Delta u_{2}\right) \\
& +\bar{\Delta}_{2}\left(x_{2}, u_{2}\right)+\nabla_{\left(x_{2}, u_{2}\right)} \hat{g}_{2}\left(x_{2}^{0}, u_{2}^{0}\right) \cdot\left(\Delta x_{2}, \Delta u_{2}\right)
\end{aligned}
$$

Based on Assumption 4, INDI control law of attitude loop is designed by using the NDI in every sampling interval

$\Delta u_{2}=\left[\nabla_{u_{2}} \hat{g}_{2}\left(x_{2}, u_{2}^{0}\right)\right]^{-1}\left(\dot{x}_{2 c}+v_{\mathrm{L} 2}-\dot{x}_{2}\right)$

$u_{2}=u_{2}^{0}+\Delta u_{2}$

where $\dot{x}_{2 c}$ is obtained from reference model designed according to the requirements of performance index, the pseudo control signal $v_{\mathrm{L} 2}$ is designed as

$v_{\mathrm{L} 2}=K_{\mathrm{P} 2}\left[\begin{array}{c}p_{c}-p \\ q_{c}-q \\ r_{c}-r\end{array}\right]+K_{\mathrm{I} 2} \int_{t_{0}}^{t}\left[\begin{array}{c}p_{c}-p \\ q_{c}-q \\ r_{c}-r\end{array}\right] \mathrm{d} \tau$

\subsection{Improved PSNN adaptive control design}

Substituting the proposed control law (18) and (22) to compound helicopter dynamics, the following closed-loop augmented compound helicopter dynamic system is derived as

$\left[\begin{array}{c}\dot{x}_{1} \\ \dot{x}_{2}\end{array}\right]=\left[\begin{array}{c}v_{\mathrm{L} 1} \\ v_{\mathrm{L} 2}\end{array}\right]+\left[\begin{array}{l}R_{1}\left(\Delta u_{1}\right)+\Delta_{1}\left(\Delta u_{1}\right) \\ R_{2}\left(\Delta u_{2}\right)+\Delta_{2}\left(\Delta u_{2}\right)\end{array}\right]$
Throughout flight envelope, even if the time interval is small, the dynamic inverse is not accurate enough when the amount of $\Delta u$ is large due to the strong nonlinearity of the control efficiency. In pure mathematical sense, that is reflected by the remarkable influence of the higher order perturbing term $R_{i}\left(\Delta u_{i}\right)$ as well as model uncertainty $\Delta_{i}\left(\Delta u_{i}\right)$. Therefore, an improved PSNN adaptive compensation is designed to overcome these undesirable influences.

According to CE principle, an adaptive term can be designed in pseudo control signal to overcome the uncertainty. Thus

$\left[\begin{array}{c}\dot{x}_{1} \\ \dot{x}_{2}\end{array}\right]=\left[\begin{array}{c}v_{\mathrm{L} 1} \\ v_{\mathrm{L} 2}\end{array}\right]+\left[\begin{array}{c}v_{a d 1} \\ v_{a d 2}\end{array}\right]+\left[\begin{array}{l}R_{1}\left(\Delta u_{1}\right)+\Delta_{1}\left(\Delta u_{1}\right) \\ R_{2}\left(\Delta u_{2}\right)+\Delta_{2}\left(\Delta u_{2}\right)\end{array}\right]$

where $v_{a d 1}$ and $v_{a d 2}$ are outputs of adaptive controller.

Remark 1 In fact, the strength of the INDI control architecture lies in the cancellation of a nonlinear and possibly multidimensional uncertainty which may include coupling of multiple states and control effects.

In this paper, The state vector $x$ and the corresponding control input $u$ and $v_{a d}$ will be used as a detailed example for design and analysis, and the same conclusions can be extended to all subsystems including $x_{1}$ and $x_{2}$. The decoupled close-loop dynamics can be obtained from (25)

$$
\begin{aligned}
\dot{x} & =\dot{x}_{c}+v_{\mathrm{L}}-v_{a d}+R(\Delta u)+\Delta(\Delta u) \\
& =\dot{x}_{c}+K_{\mathrm{P}} \tilde{x}+K_{\mathrm{I}} \int_{t_{0}}^{t} \tilde{x} \mathrm{~d} \tau-v_{a d}+\delta(\Delta u)
\end{aligned}
$$

in which $\tilde{x}$ is defined as $\tilde{x} \triangleq x_{c}-x$ and total uncertainty is defined as

$$
\begin{aligned}
\delta(\Delta u) \triangleq & R(\Delta u)+\Delta(\Delta u) \\
= & \hat{g}(x, u)-\hat{g}\left(x, u^{0}\right)-\frac{\partial \hat{g}}{\partial u}\left(u^{0}\right) \Delta u+\bar{\Delta}(x, u) \\
& -\bar{\Delta}\left(x, u^{0}\right) \\
= & \hat{g}(x, u)-\hat{g}\left(x, u^{0}\right)-\frac{\partial \hat{g}}{\partial u}\left(u^{0}\right) \Delta u+g(x, u)-\hat{g}(x, u) \\
& +\hat{g}\left(x, u^{0}\right)-g\left(x, u^{0}\right) \\
= & g(x, u)-g\left(x, u^{0}\right)-\frac{\partial \hat{g}}{\partial u}\left(u^{0}\right) \Delta u
\end{aligned}
$$

Combining (26) and (27), close-loop system with respect to state tracking error can be obtained as

$\dot{e}=A e+b\left(v_{a d}-\delta\right)$

with $e \triangleq\left[\begin{array}{c}\int_{t_{0}}^{t} \tilde{x} \mathrm{~d} \tau \\ \tilde{x}\end{array}\right] A \triangleq\left[\begin{array}{cc}0 & 1 \\ -K_{\mathrm{I}} & -K_{\mathrm{P}}\end{array}\right] b=\left[\begin{array}{l}0 \\ 1\end{array}\right]$

Assumption $5 A$ in (28) is Hurwitz.

By selecting appropriate control parameters $K_{\mathrm{P}}$ and $K_{\mathrm{I}}$, the system matrix $A$ can be made Hurwitz. Therefore, this assumption is usually satisfied. 
The closeness of the approximation is captured by the total uncertainty, which we may express in terms of the pseudo control signal as

$\delta(\Delta u)=\dot{\tilde{x}}-K_{\mathrm{P}} \tilde{x}-K_{\mathrm{I}} \int_{t_{0}}^{t} \tilde{x} \mathrm{~d} \tau+v_{a d}$

The total uncertainty $\delta$ depends on $v_{a d}$ whereas $v_{a d}$ will be designed to cancel $\delta$. This poses a fixed-point problem with existence and uniqueness of its solution $v_{a d}$ guaranteed with the following assumption

Assumption 6 The mapping $v_{a d} \rightarrow \delta$ is a contraction over the entire input domain of interest satisfied by

$$
\begin{aligned}
\left|\frac{\partial \delta(\Delta u)}{\partial v_{a d}}\right|= & \left|\frac{\partial\left[g(x, u)-g\left(x, u^{0}\right)-\frac{\partial \hat{g}}{\partial u}\left(u^{0}\right) \Delta u\right]}{\partial \Delta u}\right| \\
& \cdot\left|\frac{\partial \Delta u}{\partial\left(\dot{\tilde{x}}+v_{\mathrm{L}}-v_{a d}\right)} \frac{\partial\left(\dot{\tilde{x}}+v_{\mathrm{L}}-v_{a d}\right)}{\partial v_{a d}}\right| \\
= & \left|\frac{\partial\left[g(x, u)-g\left(x, u^{0}\right)-\frac{\partial \hat{g}}{\partial u}\left(u^{0}\right) \Delta u\right]}{\partial \Delta u}\right| \\
& \cdot\left|\frac{\partial \Delta u}{\partial\left[g\left(x, u^{0}\right)+\frac{\partial \hat{g}}{\partial u}\left(u^{0}\right) \Delta u\right]}\right|<1
\end{aligned}
$$

which can be rewritten as follows

$$
\left|\frac{\partial g / \partial \Delta u}{\partial\left[g\left(x, u^{0}\right)+\frac{\partial \hat{g}}{\partial u}\left(u^{0}\right) \Delta u\right] / \partial \Delta u}-1\right|<1
$$

Pi-Sigma neural networks is a kind of feedforward network with a single hidden layer and product units at the output layer. The weight from the hidden layer to the output layer is fixed to 1 while the weight from the input layer to the hidden layer is adjustable. The input-output map of a PSNN can be represented as

$h_{i}=\sum_{j=1}^{N} w_{i j} x_{j}$

$y=\sigma\left(\prod_{i=1}^{K} h_{i}\right)$

where $w_{i j}$ are the adjustable weights, $x_{j}$ are the scalar inputs, $K$ is the number of summing units, $N$ is the number of input nodes, $y$ is the PSNN scalar output, $h_{i}$ is the output of each summing unit, and $\sigma$ is a suitable nonlinear transfer function. For convenience define the weight vector and matrix as

$W_{i}=\left[\begin{array}{lllll}\theta_{w i} & w_{i 1} & w_{i 2} & \ldots & w_{i N}\end{array}\right]^{\mathrm{T}}$

$$
\begin{aligned}
W & =\left[\begin{array}{llll}
W_{1} & W_{2} & \cdots & W_{K}
\end{array}\right] \\
& =\left[\begin{array}{cccc}
\theta_{1} & \theta_{2} & \cdots & \theta_{K} \\
w_{11} & w_{21} & \cdots & w_{K 1} \\
\vdots & \vdots & \ddots & \vdots \\
w_{1 N} & w_{2 N} & \cdots & w_{K N}
\end{array}\right]
\end{aligned}
$$

It's also convenient to define a vector

$\bar{x}=\left[\begin{array}{lllll}b_{x} & x_{1} & x_{2} & \cdots & x_{N}\end{array}\right]^{\mathrm{T}}$

where $b_{x} \geq 0$ allows for thresholds $\theta_{w i}$ to be included in the weight vector. With the previous definitions, the input-output map of a PSNN can be written in matrix form as

$y=\sigma\left(\prod_{i=1}^{K} W_{i}^{\mathrm{T}} \bar{x}\right)$

We consider the uncertainty $\delta$ is a function mainly depending on $\Delta u$, and

$$
\begin{aligned}
\Delta u & =\left[\frac{\partial \hat{g}}{\partial u}\left(u^{0}\right)\right]^{-1}\left(\dot{x}_{c}+v_{\mathrm{L}}-v_{a d}-\dot{x}\right) \\
& =\left[\frac{\partial \hat{g}}{\partial u}\left(u^{0}\right)\right]^{-1}\left(\dot{\tilde{x}}+K_{\mathrm{P}} \tilde{x}+K_{\mathrm{I}} \int_{t_{0}}^{t} \tilde{x} \mathrm{~d} \tau-v_{a d}\right)
\end{aligned}
$$

The functional dependency of $\delta$ is

$\delta \sim \delta\left(\tilde{x}, \dot{\tilde{x}}, x, \int_{t_{0}}^{t} \tilde{x} \mathrm{~d} \tau, v_{a d}, v_{\mathrm{L}}\right)$

Therefore, PSNN input is chosen as

$\bar{x}=\left[b_{x}, \tilde{x}, \dot{\tilde{x}}, x, \int_{t_{0}}^{t} \tilde{x} \mathrm{~d} \tau, v_{\mathrm{L}}, v_{a d},\|\hat{W}\|\right]^{\mathrm{T}}$

where $\hat{W}$ is estimate of ideal weight matrix and $b_{x}$ is constant bias value.

Remark 2 Due to nonaffinity of the compound helicopter with respect to control, the total uncertainty $\delta$ is general a function of the pseudo control, including the output of the PSNN. Since the output of the PSNN is providing compensation for the interference, a fixed-point problem occurs. To ensure that Assumption 6 holds, $v_{\mathrm{L}}$ and $v_{a d}$ are chosen as inputs to the PSNN through a squashing function.

The adaptative law for the weight matrix $W$ is derived based on the Lyapunov theory. Firstly, the adaptive term constructed with the PSNN output is defined as follows

$v_{a d}=\hat{v}_{a d}+v_{r}$

$\hat{v}_{a d}=\sigma\left(\prod_{i=1}^{K} \hat{W}_{i}^{\mathrm{T}} \bar{x}\right)$

where $v_{r}$ is a term that robustifies against the effects of higher order terms in the Taylor series approximation in what follows. For convenience we define the function

$h(\hat{W}) \triangleq \sum_{i=1}^{K} \prod_{j \neq i}^{K}\left\|\hat{W}_{j}\right\|$ 


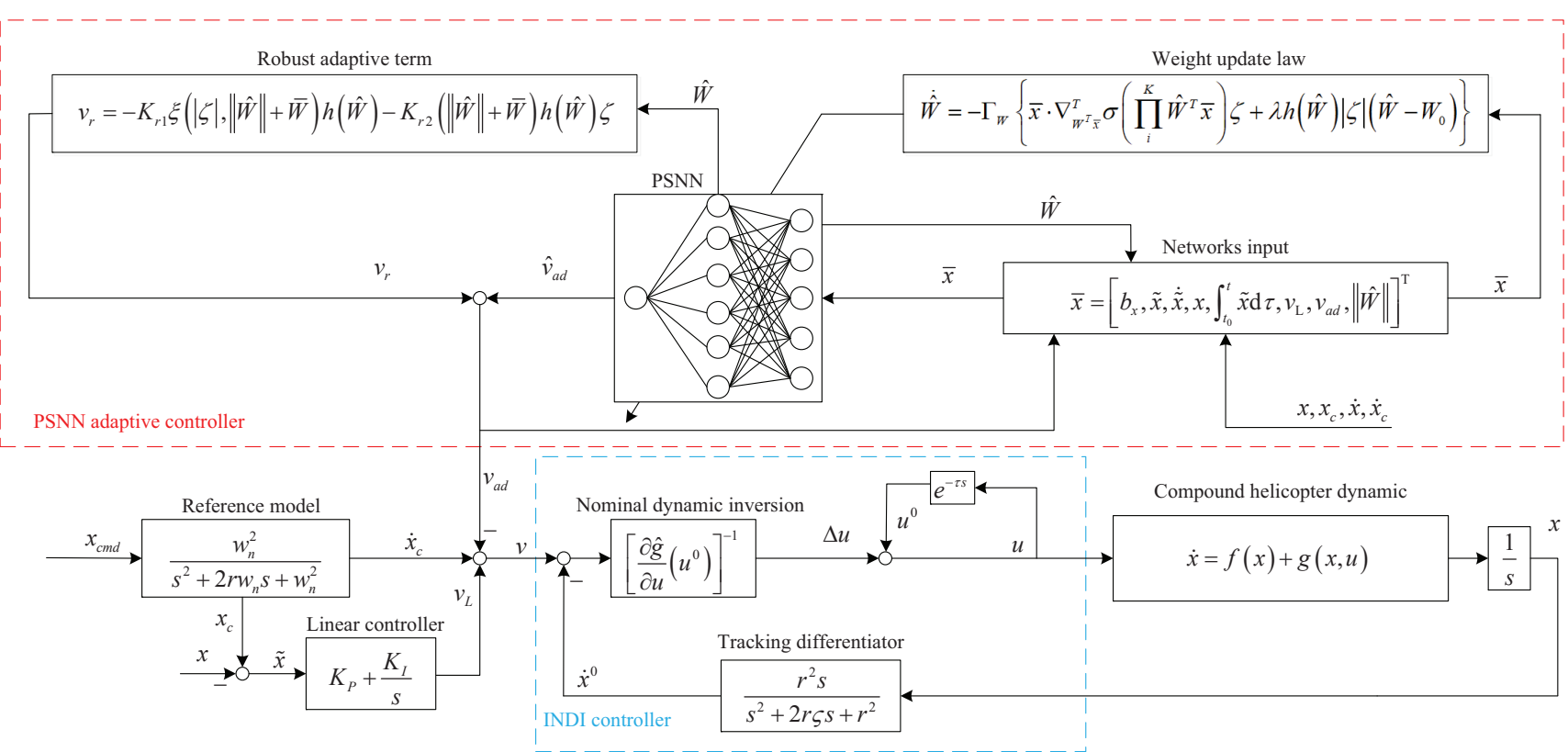

Fig. 11 Structure of close-loop system

where $h(\hat{W})$ is strictly positive due to each weight vector is designed to contain a constant bias, and $v_{r}$ is designed as

$v_{r}=-K_{r 1} \xi(|\zeta|,\|\hat{W}\|+\bar{W}) h(\hat{W})-K_{r 2}(\|\hat{W}\|+\bar{W}) h(\hat{W}) \zeta$

where $\xi(\cdot), \zeta$ and $\bar{W}$ are known terms defined later, and $K_{r 1}$, $K_{r 2}$ are robust gains. $W_{0}=\left[W_{10} W_{20} \cdots W_{K 0}\right]$ is defined as a pretrained weight matrix with $\left\|W^{*}-W_{0}\right\| \leq \bar{W}_{0}$. The update law of the weight matrix $\hat{W}$ is designed as

$\dot{\hat{W}}=-\Gamma_{W}\left[\bar{x} \cdot \nabla_{W^{T} \bar{x}} \sigma\left(\prod_{i}^{K} \hat{W}^{T} \bar{x}\right) \zeta+\lambda h(\hat{W})|\zeta|\left(\hat{W}-W_{0}\right)\right]$

where $\Gamma_{W}$ is the learning rate. The damping term $\lambda h(\hat{W}) \mid \zeta$ $\mid\left(\hat{W}-W_{0}\right)$ defined as e-modification of PSNN adaptive control is mainly used to guarantee the robustness while approximation error of the network exists.

Remark 3 Persistent excitation (PE) condition is very hard to provide for all but the simplest NNs. The e-modification corrects the potential parameter drift that may occur in the absence of PE.

The structure of the proposed close-loop system is shown in Fig. 11.

To obtain the optimal pseudo inverse in (18) and (22), we next design the actual control input. In particular, consider the following optimization problem in close-loop system

$\min u_{i} \Gamma_{i}^{-1} u_{i}$

s.t. $\quad \nabla_{u_{i}} \hat{g}_{i}\left(u_{i}^{0}\right) \Delta u_{i}=\left(\dot{\tilde{x}}_{i}+v_{L i}-v_{a d i}\right)$

where subscript $i=1$ denotes variables in velocity control loop, $i=2$ denotes variables in attitude control loop, $\Gamma_{i}$ is the allocation matrix. Based on Assumption 2, $\nabla_{u_{i}} \hat{g}_{i}\left(u_{i}^{0}\right)$ is a non-square matrix with full rank. Using Lagrange multiplier method, define a Lagrange function

$$
\begin{aligned}
H\left(\Delta u_{i}, \mu_{i}\right) \triangleq & \left(u_{i}^{0}+\Delta u_{i}\right) \Gamma_{i}^{-1}\left(u_{i}^{0}+\Delta u_{i}\right) \\
& +\mu_{i}\left[\nabla_{u_{i}} \hat{g}_{i}\left(u_{i}^{0}\right) \Delta u_{i}-\dot{\tilde{x}}_{i}-v_{\mathrm{L} i}+v_{a d i}\right]
\end{aligned}
$$

where $\mu_{i}$ is Lagrange parameter. By solving $\partial H / \partial \Delta u_{i}^{*}=0$, $\Delta u_{i}^{*}$ can be obtained as

$\Delta u_{i}^{*}=\frac{1}{2} \Gamma_{i} \nabla_{u_{i}}^{\mathrm{T}} \hat{g}_{i}\left(u_{i}^{0}\right) \mu_{i}-u_{i}^{0}$

Substuting (43) into constraint, the complete optimal control law can be given as

$$
\begin{aligned}
u_{i}^{k+1}= & \Gamma_{i} \nabla_{u_{i}}^{\mathrm{T}} \hat{g}_{i}\left(u_{i}^{k}\right)\left[\nabla_{u_{i}} \hat{g}_{i}\left(u_{i}^{k}\right) \Gamma_{i} \nabla_{u_{i}}^{\mathrm{T}} \hat{g}_{i}\left(u_{i}^{k}\right)\right]^{-1} \\
& \cdot\left[\dot{\tilde{x}}_{i}+v_{\mathrm{L} i}-v_{a d i}+\Gamma_{i} \nabla_{u_{i}} \hat{g}_{i}\left(u_{i}^{k}\right) u_{i}^{k}\right]
\end{aligned}
$$

where $k$ denotes controlling time sequence.

\subsection{Stability analysis of proposed controller}

Consider a PSNN approximation of uncertainty

$\sigma\left(\prod_{i=1}^{K} W_{i}^{* T} \bar{x}\right)=\delta-\varepsilon$

where $W_{i}^{*} \in \mathbb{R}^{n}$ are column vectors of ideal weight matrix $W^{*} \in \mathbb{R}^{n \times K}$. The scalar $\varepsilon$ is referred to as the NN reconstruction error, or residual error, where $0 \leq\|\varepsilon\| \leq \bar{\varepsilon}$ and $\bar{\varepsilon}$ is an upper bound defined in what follows. The basis function $\sigma$ 
serving to approximate uncertainty $\delta$ selects the following hyperbolic tangent function.

$\sigma(z)=k_{N N} \tanh (z)$

where $k_{N N}$ is the network gain coefficient, $z \in \mathbb{R}$. PSNN input vector $\bar{x} \in \mathbb{R}^{n}$ is made up of selected elements of the states and pseudo control signals. For analytical purposes, ideal weight matrix $W^{*}=\left[W_{1}^{*} \cdots W_{K}^{*}\right]$ is defined as the value of adaptable weight $W$ over $\Omega_{W}$ that minimizes $|\varepsilon|$ for all $\bar{x} \in \Omega_{x}$ such that

$W^{*} \stackrel{\Delta}{=} \arg \min _{W \in \Omega_{W}}\left\{\sup _{\bar{x} \in \Omega_{x}}\left|\sigma\left(\prod_{i}^{K} W_{i}^{\mathrm{T}} \bar{x}\right)-\delta\right|\right\}$

And upper bound $\bar{\varepsilon}$ is

$\bar{\varepsilon} \triangleq \sup _{\bar{x} \in \Omega_{x}}\left|\sigma\left(\prod_{i}^{K} W_{i}^{* T} \bar{x}\right)-\delta\right|$

The Frobenius norm of ideal PSNN weight matrix is bounded by a known positive value

$\left\|W^{*}\right\| \leq \bar{W}$

and

$$
\|\hat{W}\|=\left\|W^{*}+\tilde{W}\right\| \leq \bar{W}+\|\tilde{W}\|
$$$$
\left\|\hat{W}_{i}\right\|=\left\|W_{i}^{*}+\tilde{W}_{i}\right\| \leq \bar{W}+\left\|\tilde{W}_{i}\right\|
$$

where $\tilde{W} \triangleq \hat{}=\hat{W}-W^{*}$ and $\tilde{W}_{i} \triangleq \hat{W}_{i}-W_{i}^{*}$ are weight estimation errors.

Using a Taylor series expansion about the current estimate of the output, the approximation error can be expressed as

$$
\begin{aligned}
\sigma\left(\prod_{i=1}^{K} W_{i}^{* \mathrm{~T}} \bar{x}\right)= & \sigma\left(\prod_{i=1}^{K} \hat{W}_{i}^{\mathrm{T}} \bar{x}\right)-\nabla_{W^{\mathrm{T}} \bar{x}} \sigma\left(\prod_{i=1}^{K} \hat{W}_{i}^{\mathrm{T}} \bar{x}\right) \\
& \cdot \tilde{W}^{\mathrm{T}} \bar{x}+o\left(\tilde{W}^{\mathrm{T}} \bar{x}\right)^{2}
\end{aligned}
$$

where

$$
\begin{aligned}
\nabla_{W^{\mathrm{T}} \bar{x}} \sigma\left(\prod_{i=1}^{K} \hat{W}_{\mathrm{i}}^{\mathrm{T}} \bar{x}\right)= & \sigma^{\prime}\left(\prod_{i=1}^{K} \hat{W}_{i}^{\mathrm{T}} \bar{x}\right) \\
& \cdot\left[\prod_{j \neq 1}^{K} \hat{W}_{j}^{\mathrm{T}} \bar{x} \prod_{j \neq 2}^{K} \hat{W}_{j}^{\mathrm{T}} \bar{x} \cdots \prod_{j \neq K}^{K} \hat{W}_{j}^{\mathrm{T}} \bar{x}\right]
\end{aligned}
$$

which can be bounded by

$$
\left\|\nabla_{W^{T} \bar{x}} \sigma\left(\prod_{i=1}^{K} \hat{W}_{i}^{\mathrm{T}} \bar{x}\right)\right\| \leq \sigma^{\prime}\left(\prod_{i=1}^{K} \hat{W}_{i}^{\mathrm{T}} \bar{x}\right) \cdot K\|\bar{x}\|^{K-1} h(\hat{W})
$$

Thus, the higher order residual term is given by

$$
\begin{aligned}
o\left(\tilde{W}^{\mathrm{T}} \bar{x}\right)^{2}= & -\nabla_{W^{\mathrm{T}} \bar{x}} \sigma\left(\prod_{i=1}^{K} \hat{W}_{i}^{\mathrm{T}} \bar{x}\right) \cdot \tilde{W}^{\mathrm{T}} \bar{x}+\sigma\left(\prod_{i=1}^{K} W_{i}^{* \mathrm{~T}} \bar{x}\right) \\
& -\sigma\left(\prod_{i=1}^{K} \hat{W}_{i}^{\mathrm{T}} \bar{x}\right)
\end{aligned}
$$

Using (48), the NN output is bounded by

$$
\left\|\hat{v}_{a d}\right\|<\sigma\left[c^{\prime} \prod_{i=1}^{K}\left(\bar{W}+\left\|\tilde{W}_{i}\right\|\right)\right]
$$

where $c^{\prime}>0$. The design as in (38) and Assumption 3 imply that the NN input can be maximally bounded in terms of the tracking performance by

$\|\bar{x}\| \leq c_{0}^{\prime}+c_{1}^{\prime}|\zeta|+c_{2}^{\prime}\|\tilde{W}\|$

The scalar $\zeta$ is filtered error term

$\zeta=e^{T} P b$

where matrix $P$ is the solution to the Lyapunov equation $A^{\mathrm{T}} P+P A=-Q$ and $P=P^{\mathrm{T}}>0$ The construction of $\zeta$ in (54) can be seen as an error filter. From Cauchy-Schwarz inequality and the compatibility of the Frobenius norm with the vector 2-norm, it is clear that

$|\zeta| \leq\|e\|\|P\|$

Using design of PSNN input (38), we have

$$
\begin{aligned}
\|\bar{x}\|^{K} & \left.\leq\left(c^{\prime}{ }_{0}+c^{\prime}{ }_{1}|\zeta|+c^{\prime}{ }_{2}\|\tilde{W}\|\right)\right)^{K} \\
& \triangleq c_{0}^{\prime}{ }_{0}-1 \\
& \left.=c^{\prime}{ }_{3}{ }^{\prime}+c^{\prime}{ }_{4}{ }_{4}|\zeta|+c^{\prime}{ }_{1}|\zeta|+c^{\prime}{ }_{5}\|\tilde{W}\|+\tilde{W} \|\right)+\xi(|\zeta|,\|\tilde{W}\|)
\end{aligned}
$$

where $c_{i}^{\prime}>0, \xi(a, b)$ is defined as a polynomial function of $a$ and $b$.

From (51) the high order term is bounded by

$\left|o\left(\tilde{W}^{T} \bar{x}\right)^{2}\right|<c_{6}^{\prime}+c_{7}^{\prime}\|\bar{x}\|\|\tilde{W}\| \cdot h(\hat{W})$

where $c_{i}^{\prime}>0$. Let $w_{p}$ be defined as the $\mathrm{NN}$ approximation error plus the higher order effects of back propagation through the PSNN

$w_{p}=-o\left(\tilde{W}^{T} \bar{x}\right)^{2}+\varepsilon$

Consider the properties of the PSNN structure and (56), an upper bound on $w_{p}$ is

$\left|w_{p}\right|<\varepsilon+c_{6}^{\prime}+c_{7}^{\prime}\|\bar{x}\|^{K}\|\tilde{W}\| h(\hat{W})$ 
Combine this with (54), then

$$
\begin{aligned}
\left|w_{p}\right| & <\varepsilon+c^{\prime}{ }_{6}+c^{\prime}{ }_{7}\|\bar{x}\|^{K}\|\tilde{W}\| h(\hat{W}) \\
& \leq \varepsilon+c^{\prime}{ }_{6}+c^{\prime}{ }_{7}\left(c^{\prime}{ }_{0}+c^{\prime}{ }_{1}|\zeta|+c^{\prime}{ }_{2}\|\tilde{W}\|\right)^{K} \cdot\|\tilde{W}\| h(\hat{W}) \\
& =\varepsilon+c^{\prime}{ }_{6}+c^{\prime}{ }_{7}\left\{c^{\prime}{ }_{3}+c^{\prime}{ }_{4}|\zeta|+c^{\prime}{ }_{5}\|\tilde{W}\|+\xi(|\zeta|,\|\tilde{W}\|)\right\} \\
& \cdot\|\tilde{W}\| h(\hat{W}) \\
& =c_{0}+c_{1}\|\tilde{W}\| h(\hat{W})+c_{2}|\zeta|\|\tilde{W}\| h(\hat{W})+c_{3}\|\tilde{W}\|^{2} h(\hat{W}) \\
& +c_{4} \xi(|\zeta|,\|\tilde{W}\|) h(\hat{W})
\end{aligned}
$$

where $c_{i}>0$ are known.

Theorem 1 Consider the compound helicopter nonlinear dynamics given by (12) together with the control law given by (18) and (22), and adaptive law given by (41) satisfying assumptions 1-6 as well as the following condition

$$
\left\{\begin{array}{c}
\Gamma_{W}>0 \\
K_{\mathrm{r} 1} \geq c_{4} \\
K_{\mathrm{r} 2} \geq c_{2} \\
c_{3}+\frac{c_{1}}{2 \sqrt{c_{0}}} \geq \lambda \geq c_{3}
\end{array}\right.
$$

Such that for initial conditions $x(0), \tilde{W}(0)$ belonging to compact set $\Omega_{0}$, it can be guaranteed that all the signals in the proposed closed-loop system are semi-globally uniformly ultimately bounded (SGUUB) and the tracking error $e(t)$ and the weight error $\tilde{W}(t)$ converge to the zero point.

Proof of theorem 1 The tracking error dynamics are given by

$$
\begin{aligned}
\dot{e}= & A e+b\left(v_{a d}-\delta\right)=A e \\
& +b\left\{v_{r}+\sigma\left(\prod_{i=1}^{K} W_{i}^{\mathrm{T}} \bar{x}\right)-\sigma\left(\prod_{i=1}^{K} W_{i}^{* \mathrm{~T}} \bar{x}\right)-\varepsilon\right\}
\end{aligned}
$$

Consider the candidate Lyapunov function

$V(e(t), \tilde{W}(t))=\frac{1}{2} e^{T} P e+\frac{1}{2 \Gamma_{W}} \operatorname{tr}\left(\tilde{W}^{T} \tilde{W}\right)$

Combining with the Lyapunov function $A^{T} P+P A=-Q$ for $Q=Q^{\mathrm{T}}>0$ and substituting the update law, the derivative of the Lyapunov function with respect to time of this expres- sion may be expressed as

$$
\begin{aligned}
\dot{V} & (e(t), \tilde{W}(t)) \\
= & \frac{1}{2} e^{\mathrm{T}} P\left[A e+b\left(v_{a d}-\delta\right)\right]+\frac{1}{2}\left[A e+b\left(v_{a d}-\delta\right)\right]^{\mathrm{T}} P e \\
& +\operatorname{tr}\left\{\tilde{W}^{\mathrm{T}} \Gamma_{W}^{-1} \dot{\tilde{W}}\right\} \\
= & e^{\mathrm{T}}\left(A^{\mathrm{T}} P+P A\right) e+\left(v_{a d}-\delta\right) b^{\mathrm{T}} P e+\operatorname{tr}\left\{\tilde{W}^{\mathrm{T}} \Gamma_{W}^{-1} \dot{\tilde{W}}\right\} \\
= & \left\{\sigma\left(\prod_{i=1}^{K} \hat{W}_{i}^{\mathrm{T}} \bar{x}\right)+v_{r}-\sigma\left(\prod_{i=1}^{K} W_{i}^{* \mathrm{~T}} \bar{x}\right)+\varepsilon\right\} \zeta \\
& -e^{\mathrm{T}} Q e+\operatorname{tr}\left\{\tilde{W}^{\mathrm{T}} \Gamma_{W}^{-1} \dot{\tilde{W}}\right\} \\
= & \left\{v_{r}+\nabla_{W^{\mathrm{T}} \bar{x}} \sigma\left(\prod_{i=1}^{K} \hat{W}_{i}^{\mathrm{T}} \bar{x}\right) \cdot \tilde{W}^{\mathrm{T}} \bar{x}-o\left(\tilde{W}^{\mathrm{T}} \bar{x}\right)^{2}+\varepsilon\right\} \zeta \\
& +\operatorname{tr}\left\{\tilde{W}^{\mathrm{T}} \Gamma_{W}^{-1} \dot{\tilde{W}}^{\dot{W}^{2}}-e^{\mathrm{T}} Q e\right. \\
= & \left\{v_{r}-o\left(\tilde{W}^{T} \bar{x}\right)^{2}+\varepsilon\right\} \zeta-e^{\mathrm{T}} Q e \\
& +\operatorname{tr}\left\{\tilde{W}^{\mathrm{T}}\left[\bar{x} \cdot \nabla_{W^{\mathrm{T}} \bar{x}} \sigma\left(\prod_{i=1}^{K} \hat{W}_{i}^{\mathrm{T}} \bar{x}\right) \zeta+\Gamma_{W}^{-1} \dot{\tilde{W}}^{\prime}\right]\right\} \\
= & -e^{\mathrm{T}} Q e+\left\{v_{r}+w_{p}(\bar{x}, \tilde{W})\right\} \xi \\
& -\lambda|\zeta| h(\hat{W}) \operatorname{tr}\left\{\tilde{W}^{\mathrm{T}}\left(\hat{W}-W_{0}\right)\right\}
\end{aligned}
$$

Use the known bound $\left\|W^{*}-W_{0}\right\| \leq \bar{W}_{0}$, Cauchy-Schwarz inequality and note that

$$
\begin{aligned}
& -\operatorname{tr}\left\{\tilde{W}^{\mathrm{T}}\left(\hat{W}-W_{0}\right)\right\}=-\operatorname{tr}\left\{\tilde{W}^{\mathrm{T}}\left(\tilde{W}+W^{*}-W_{0}\right)\right\} \\
& =-\|\tilde{W}\|^{2}-\operatorname{tr}\left\{\tilde{W}^{\mathrm{T}}\left(W^{*}-W_{0}\right)\right\} \\
& =-\|\tilde{W}\|^{2}-\sum_{i=1}^{K} \tilde{W}_{i}^{\mathrm{T}}\left(W_{i}^{*}-W_{i 0}\right) \\
& \leq-\|\tilde{W}\|^{2}+\|\tilde{W}\| \bar{W}_{0}
\end{aligned}
$$

For convenience of analysis, choose $Q=I$. Observe that

$$
e^{\mathrm{T}} P e \leq \rho(P)\|e\|^{2} \Rightarrow-e^{\mathrm{T}} e=-\|e\|^{2} \leq-\frac{e^{\mathrm{T}} P e}{\rho(P)}
$$


where $\rho(P)$ signifies the spectral radius of positive-definite matrix. Therefore

$$
\begin{aligned}
& \dot{V}(e(t), \tilde{W}(t)) \\
& \leq\left\{v_{r}+w_{p}(\bar{x}, \tilde{W})\right\} \xi-\lambda h(\hat{W})|\zeta| \operatorname{tr}\left\{\tilde{W}^{\mathrm{T}} \tilde{W}\right\}-\frac{e^{\mathrm{T}} P e}{\rho(P)} \\
& -\lambda h(\hat{W})|\zeta| \operatorname{tr}\left\{\tilde{W}^{\mathrm{T}}\left(\tilde{W}+W^{*}-W_{0}\right)\right\} \\
& \leq v_{r} \zeta-\lambda\|\tilde{W}\|^{2} h(\hat{W})|\zeta|-\frac{e^{\mathrm{T}} P e}{\rho(P)}+\lambda \bar{W}_{0}\|\tilde{W}\| h(\hat{W})|\zeta| \\
& +\sup \left\{w_{p}(\bar{x}, \tilde{W})\right\} \zeta \\
& =-\frac{e^{\mathrm{T}} P e}{\rho(P)}-\lambda|\zeta|\|\tilde{W}\|^{2} h(\hat{W})+\lambda|\zeta| \bar{W}_{0}\|\tilde{W}\| h(\hat{W}) \\
& -K_{r 1} \xi\left(|\zeta|,\|\hat{W}\|+\bar{W}_{0}\right) h(\hat{W}) \zeta-K_{r 2}\left(\|\hat{W}\|+\bar{W}_{0}\right) \\
& \cdot h(\hat{W}) \zeta^{2}+\left\{c_{0}+c_{1}\|\tilde{W}\| h(\hat{W})+c_{2}|\zeta|\|\tilde{W}\| h(\hat{W})\right. \\
& \left.+c_{3}\|\tilde{W}\|^{2} h(\hat{W})+c_{4} \xi(|\zeta|,\|\tilde{W}\|) h(\hat{W})\right\} \zeta \\
& \leq-\frac{e^{\mathrm{T}} P e}{\rho(P)}+c_{0}|\zeta|+\left(c_{1}+\lambda \bar{W}_{0}\right)|\zeta|\|\tilde{W}\| h(\hat{W}) \\
& +\left(c_{2}-K_{r 2}\right)\|\tilde{W}\| h(\hat{W}) \zeta^{2}+\left(c_{3}-\lambda\right)\|\tilde{W}\|^{2} h(\hat{W})|\zeta| \\
& +\left(c_{4}-K_{r 1}\right) \xi\left(|\zeta|,\|\hat{W}\|+\bar{W}_{0}\right) h(\hat{W}) \zeta
\end{aligned}
$$

With the following condition

$$
\left\{\begin{array}{l}
\Gamma_{W}>0 \\
K_{r 1} \geq c_{4} \\
K_{r 2} \geq c_{2}
\end{array}\right.
$$

such that

$$
\begin{aligned}
\dot{V}(e(t), \tilde{W}(t)) \leq & -\frac{e^{\mathrm{T}} P e}{\rho(P)}+\left\{c_{0}+\left(c_{1}+\lambda \bar{W}_{0}\right)\|\tilde{W}\|\right. \\
& \left.+\left(c_{3}-\lambda\right)\|\tilde{W}\|^{2}\right\} h(\hat{W})|\zeta|
\end{aligned}
$$

with $\lambda>c_{3}$ this can be written as a quadratic form

$$
\begin{aligned}
\dot{V}(e(t), \tilde{W}(t)) & \\
\leq & -\frac{e^{\mathrm{T}} P e}{\rho(P)}+\left\{\left(c_{3}-\lambda\right)\left[\|\tilde{W}\|-\frac{\left(c_{1}+\lambda \bar{W}_{0}\right)}{2\left(c_{3}-\lambda\right)}\right]^{2}\right. \\
& \left.-\frac{\left(c_{1}+\lambda \bar{W}_{0}\right)^{2}}{4\left(c_{3}-\lambda\right)^{2}}+c_{0}\right\} h(\hat{W})|\zeta|
\end{aligned}
$$

when $\lambda$ satisfies the condition that

$c_{3}+\frac{c_{1}}{2 \sqrt{c_{0}}} \geq \lambda>c_{3}$

thus

$\frac{\left(c_{1}+\lambda \bar{W}_{0}\right)^{2}}{4\left(c_{3}-\lambda\right)^{2}}>\frac{c_{1}^{2}}{4\left(c_{3}-\lambda\right)^{2}} \geq c_{0}$

which implies that $\dot{V}$ will be negative with each term in the formula is strictly negative, thus the error $e$ and the weight
Table 1 Simulation model main parameters

\begin{tabular}{ll}
\hline Parameter & Value \\
\hline Gross weight & $7438 \mathrm{~kg}$ \\
VTDP rotor speed & $2160 \mathrm{rpm}$ \\
Lift curve slope & 5.73 \\
Main rotor inertia & $1512.6 \mathrm{~kg} \cdot \mathrm{m}^{2}$ \\
Wings dihedral angle & $7^{\circ}$ \\
Initial angle of attack & $6^{\circ}$ \\
Sweptback angle & $11.375^{\circ}$ \\
Hinge offset & 0.04659 \\
Blade twist & -0.3142 \\
Longitudinal inclination & $3^{\circ}$ \\
Blade lock number & 8.19 \\
Main rotor radius & $8.18 \mathrm{~m}$ \\
Main rotor chord & $0.527 \mathrm{~m}$ \\
Main rotor speed & $258 \mathrm{rpm}$ \\
Blade stiffness & $0.08 \mathrm{~N} / \mathrm{m}$ \\
Forward tilting angle of rotor shaft & $3^{\circ}$ \\
\hline
\end{tabular}

Table 2 Initial parameters setting in proposed controller

\begin{tabular}{llll}
\hline Parameter & Value & Parameter & Value \\
\hline$w_{0}$ & 0.01 & $\theta_{w i}$ & 0.02 \\
$b_{w}$ & 0.1 & $\Gamma_{w}$ & 100 \\
$\lambda$ & 0.6 & $K_{r 1}$ & 1.4 \\
$K_{r 2}$ & 1.28 & $b_{x}$ & 0.1 \\
$\bar{W}$ & 5 & $k_{N N}$ & 1.2 \\
\hline
\end{tabular}

estimation error $\tilde{W}$ will converge to the zero point. All the signals in the closed-loop system are proved SGUUB.

Remark 4 Note that $\lambda, \Gamma_{W}, K_{r 1}$ and $K_{r 2}$ are the design parameters and can determine a trade-off between control performance and robustness. The external command signals were assumed to be bounded, as in assumption 2. This shows that commands of larger magnitude imply smaller values for $\Gamma_{W}$. This may be interpreted to mean that to limit the closed-loop bandwidth, smaller PSNN learning rates may be required when allowing for more aggressive command tracking.

\section{Numerical simulation}

In this section, numerical examples are provided to demonstrate the effectiveness and improved performance of the proposed approach.

The proposed approach is designed to solve a class of compound helicopter automatic control problem. In this study, $\mathrm{X}-49$ known as a classical compound helicopter is taken as an example in this simulation. The main rotor and the fuselage's non-linear mathematical models are built using data from General Helicopter (GENHEL) Flight Dynamics Simulation. The main parameters are shown in the table below. An approximate nominal nonlinear model $\hat{g}_{i}(x, u)$ is used to get incremental dynamic inversion. The nominal model is established by combining known parts of each component 
model and ignoring random aerodynamic interference, control surface coupling as well as model uncertainty varying with flight state, which are considerable and may cause fatal instability during actual flight.

The initial state is set to hovering equilibrium state, of which all states set to zero. The forward flight command $u_{c m d}$ is given by as a multimodal maneuver signal, which contains two aggressive maneuver processes and two cruise processes.

$u_{c m d}(t)=\left\{\begin{array}{cc}36.7 t & t<30 s \\ 110 & 30 s<t<50 s \\ 610-10 t & 50 s<t<55 s \\ 60 & 55 s<t\end{array}\right.$

Furthermore, realistic strong aerodynamic cross-coupling among various control surfaces is designed through the experimental data to make it more difficult for the controller to keep the compound helicopter stable. The velocity reference model is designed as an inertial link

$x_{1 c}(s)=\frac{1}{0.3 s+1} x_{1 c m d}(s)$

where all parameters in reference are designed according to performance index. The velocity optimization matrix is designed as

$\Gamma_{1}=\operatorname{diag}(1,1,2,5000)$

To ensure Assumption 4 and reduce system burden, the short time increment $T_{s}=20 \mathrm{~ms}$. Designed as (44), the velocity control input recursion is

$$
\begin{aligned}
u_{1}^{k+1}= & \Gamma_{1} \nabla_{u_{1}}^{\mathrm{T}} \hat{g}_{1}\left(x, u_{1}^{k}\right)\left[\nabla_{u_{1}} \hat{g}_{1}\left(x, u_{1}^{k}\right) \Gamma_{1} \cdot \nabla_{u_{1}}^{\mathrm{T}} \hat{g}_{1}\left(x, u_{1}^{k}\right)\right]^{-1} \\
& {\left[\dot{\tilde{x}}_{1}+v_{\mathrm{L} 1}-v_{a d 1}+\nabla_{u_{1}} \hat{g}_{1}\left(x, u_{1}^{k}\right) u_{1}^{k}\right] }
\end{aligned}
$$

Similarly, the angular velocity reference model is designed as a second order system.

$x_{2 c}(s)=\frac{17.64}{s^{2}+8.4 s+17.64} x_{2 c m d}(s)$

The optimization matrix is

$\Gamma_{2}=\operatorname{diag}(0.33,1,1.25,1.25,2,0.66)$

Attitude control input recursion is

$$
\begin{aligned}
u_{2}^{k+1}= & \Gamma_{2} \nabla_{u_{2}}^{\mathrm{T}} \hat{g}_{2}\left(x, u_{2}^{k}\right)\left[\nabla_{u_{2}} \hat{g}_{2}\left(x, u_{2}^{k}\right) \Gamma_{2} \cdot \nabla_{u_{2}}^{\mathrm{T}} \hat{g}_{2}\left(x, u_{2}^{k}\right)\right]^{-1} \\
& {\left[\dot{\tilde{x}}_{2}+v_{\mathrm{L} 2}-v_{a d 2}+\nabla_{u_{2}} \hat{g}_{2}\left(x, u_{2}^{k}\right) u_{2}^{k}\right] }
\end{aligned}
$$

where $u_{i}^{0}$ is initial control input, the structure of pseudo control $v_{\mathrm{L} i}$ and $v_{a d i}$ is given before, the detailed control parameters are elaborately tuned as table below. To ensure the accuracy of feedback differential signals, the second order tracking differentiator is used to remove noise as

$y(s)=\frac{s}{2.4 s^{2}+3.8 s+1} v(s)$ (a)

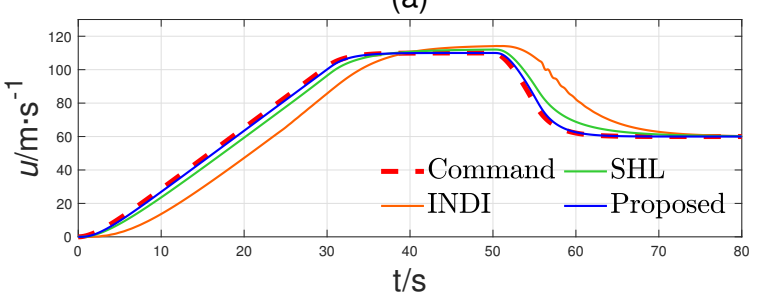

(b)

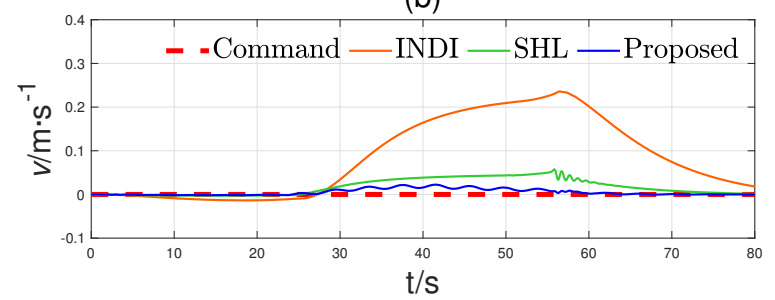

(c)

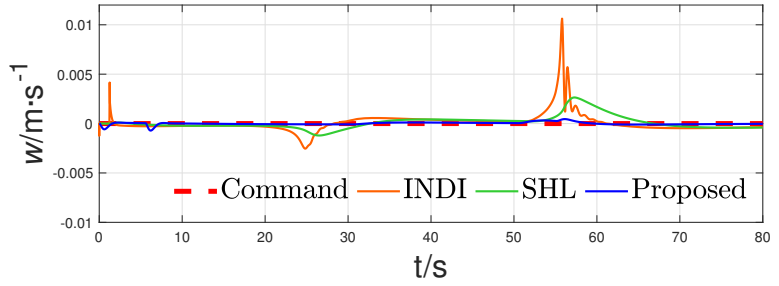

Fig. 12 Time responses of the velocity state variables of compound helicopter

During the simulation, forth order PSNN including 4 hidden neurons and 32 adjustable weights is selected in both velocity tracking control and angle velocity tracking control. The input of neural network is designed in (38). Used as controls, the INDI controller without adaptive compensation and single layer perceptron (SHL) neural networks adaptive controller combined with INDI including 10 hidden neurons and 90 adjustable weights are built in the simulation. The key parameters including learning rates in both two networks are designed to be the same.

The simulation process is carried out in MATLAB, and the results are shown in figures below. Fig.12(a) shows that proposed controller successfully tracked desired forward velocity command and made it possible to improve tracking performance by designing reference model according to performance index of time domain or frequency domain. Nevertheless, INDI controller and SHL adaptive controller only followed the command barely. Fig.12(b)-Fig.14 show convergence performance related to attitude and velocity. It can be observed that attitude, angular velocity and velocity errors driven by proposed control scheme asymptotically converge to origin even though flight state rapid change, dynamics inaccuracy, as well as aerodynamic coupling interference. However, in contrast, the other two methods just 
(a)

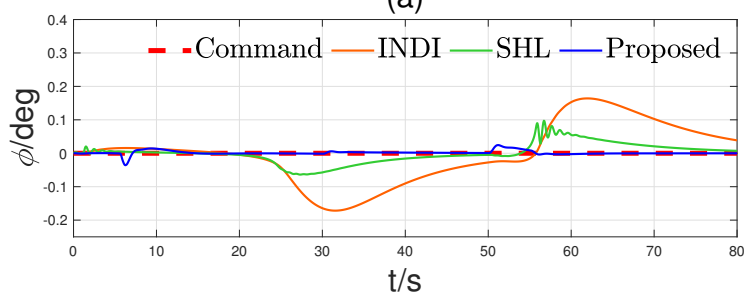

(b)

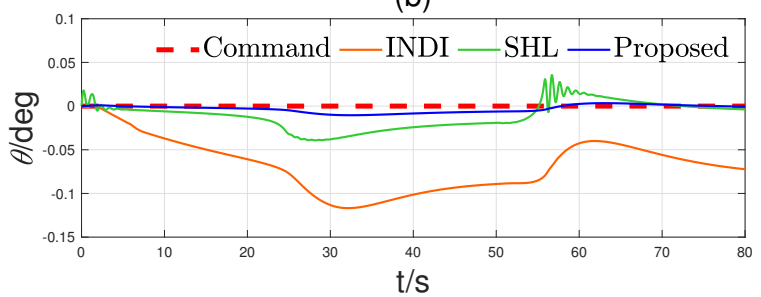

(c)

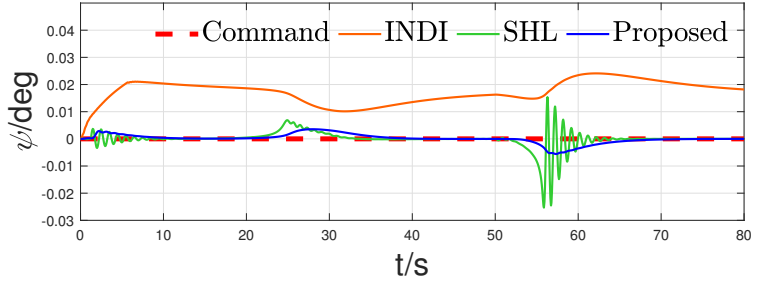

Fig. 13 Time responses of the Euler angle state variables of compound helicopter

guarantee bounded results within simulation time and can't eliminate violent shock during the maneuver process.

Fig.15 and Fig.16 show that proposed controller ensures that multiple redundant control surfaces can complete the maneuver task in an efficient way during the whole maneuver process of the compound helicopter, and guarantee the smoothness of mode conversion. The helicopter control surfaces mainly work in low-speed flight and keep minimum mode in high-speed flight. On the contrary, fixed wing control surfaces play important roles in high-speed flight. Fig.17 and Fig. 18 show that proposed controller relied on the output of linear controller at first, while the adaptive controller realized the cancellation of perturbation term and convergence of error in a short time (about $10 \mathrm{sec}$ ), and had a vital catalytic role in the process of maintaining stability. It can also be concluded that as well as tracking error tend to be flat due to PSNN. Along with the flight states varying, the PSNN adaptive controller output changed fast enough to maintain robustness to disturbances, which is superior to the traditional neural networks.

Fig.19 and Fig.20 shows that weight matrix norms converge in 10 second with great robustness. Although in the process of network convergence, the weight produced inevitable oscillation phenomenon, the robust adaptive term (a)

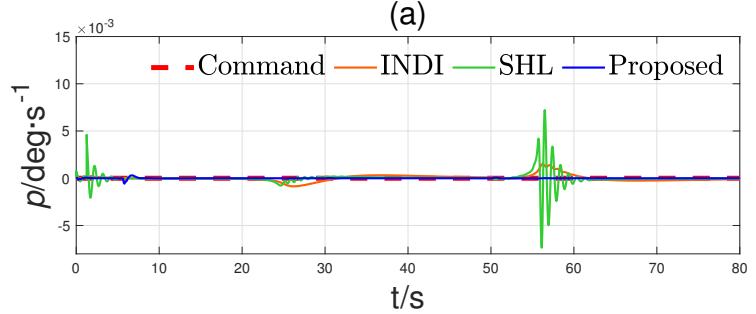

(b)

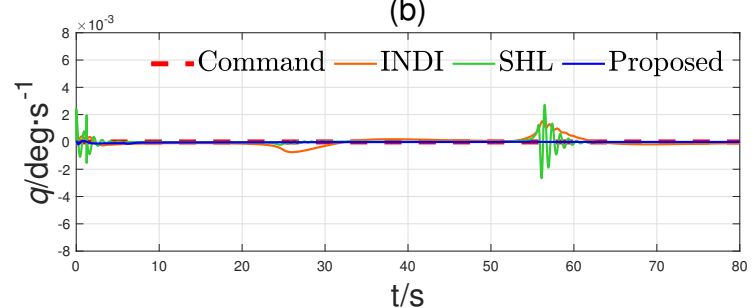

(c)

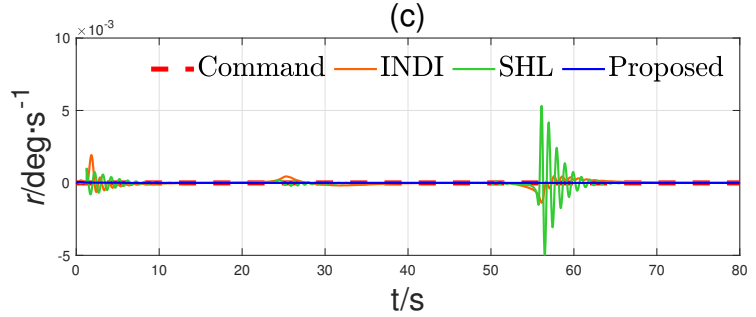

Fig. 14 Time responses of the angular velocity state variables of compound helicopter
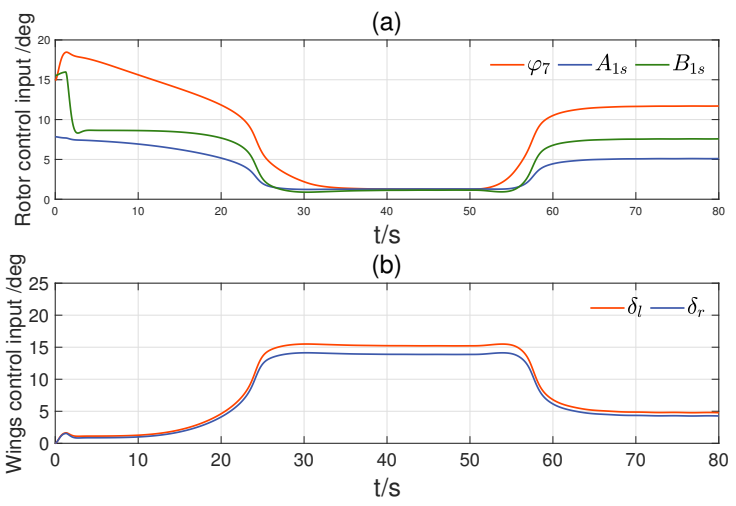

Fig. 15 Time histories for control surfaces including $\varphi_{7}, A_{1 s}, B_{1 s}, \delta_{l}$ and $\delta_{r}$

designed in (40) and the damping item designed in (41) still helped keep PSNN stable. During first maneuver process, the convergence of weights was completed after a series of small shocks. Moreover, during second maneuver process, although the process is more violent, the norm of weight matrix still maintained a steady process, which implies that the uncertain nonlinear perturbation has been successfully cancelled by PSNN and local minimum has been successfully avoided. In engineering, storing the PSNN parameters 


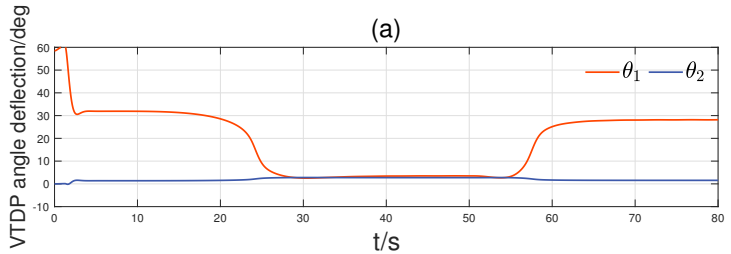

(b)

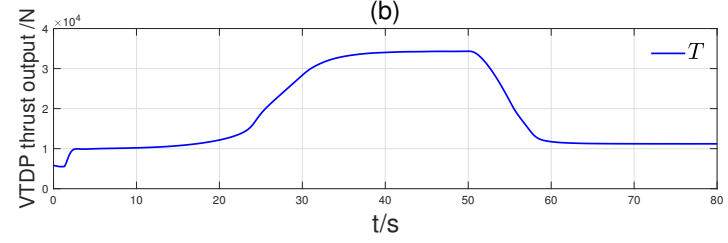

Fig. 16 Time histories for control surfaces including $\theta_{1}, \theta_{2}$ and $T$

(a)

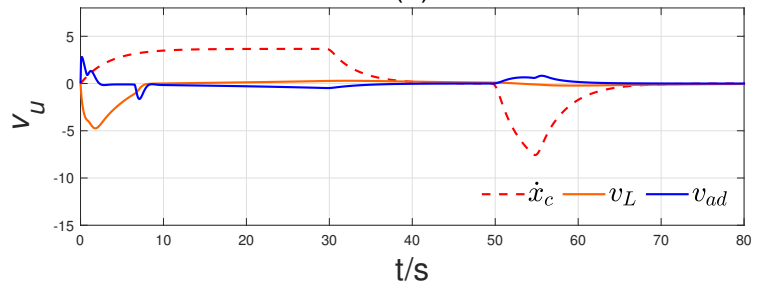

(b)

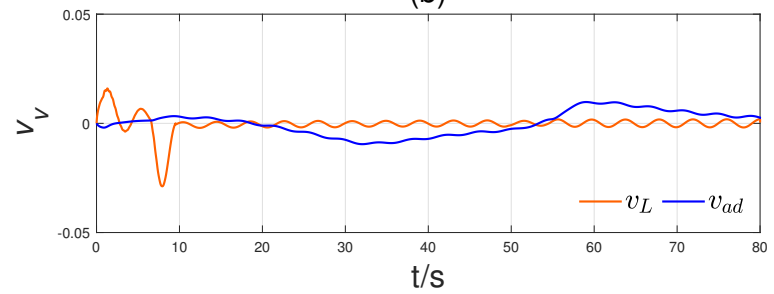

(c)

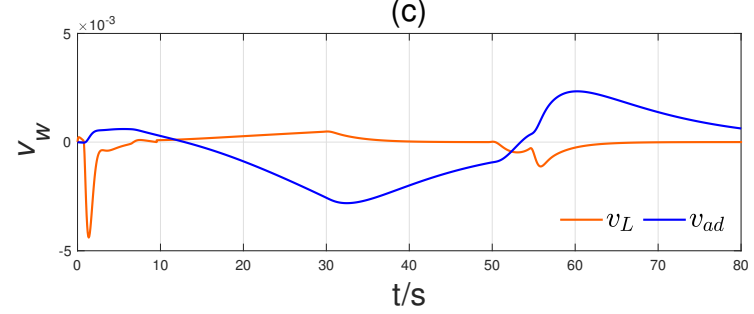

Fig. 17 Time histories for all pseudo velocity control signals obtained from reference model, linear controller and PSNN adaptive controller

can further improve the stability and robustness of the closeloop system.

\section{Conclusion}

By incorporating adaptive control method into PSNN controller design, the proposed novel method with global stability, fast convergence ability and strong robustness have been proposed for solving command tracking problem of compound helicopters with aerodynamic cross-coupling in (a)

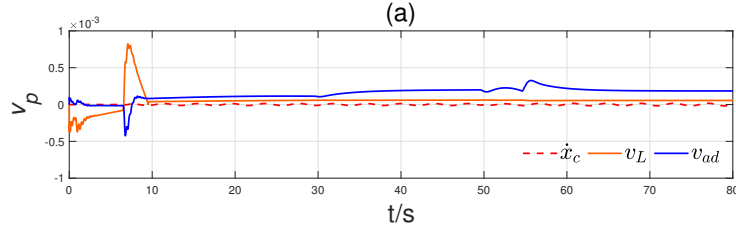

(b)

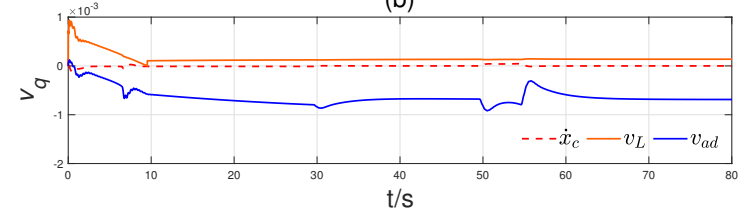

(c)

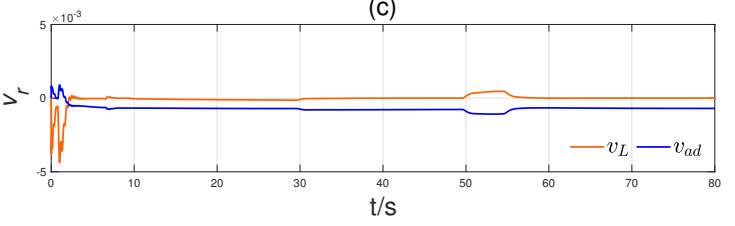

Fig. 18 Time histories for all pseudo angular velocity control signals obtained from reference model, linear controller and PSNN adaptive controllery

(a)

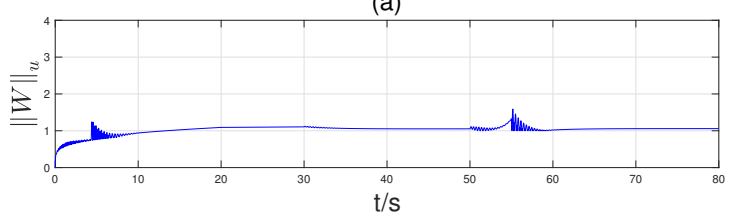

(b)

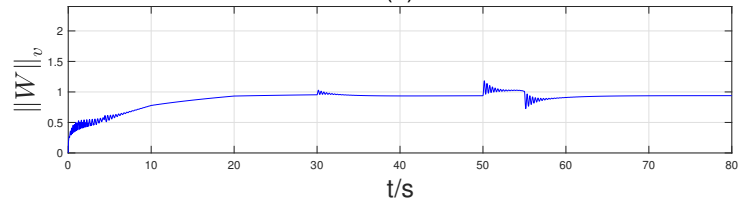

(c)

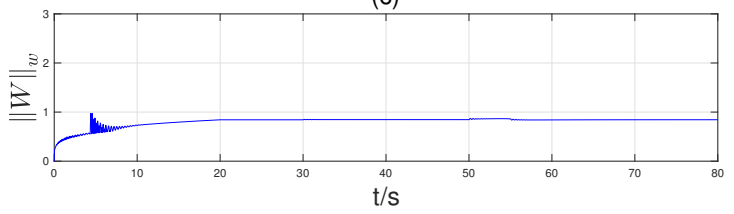

Fig. 19 Time histories for Frobenius norm of weight matrices to velocity control

multimodal maneuver. The weights of PSNN are adjusted online according to a novel adaptive algorithm based on $\mathrm{CE}$ principle such that the effects of the uncertain coupling disturbance can be mitigated under the condition of close-loop augmented system SGUUB. INDI control framework including a couple of control loop has been built to decouple the overactuated system and keep robustness to unstable characteristic of PSNN. Moreover, on the basis of an incremental Lagrange multiplier optimization, the globally optimal input for each control surface is allocated based on its time- 
(a)

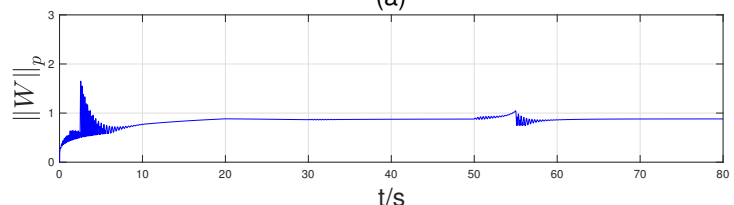

(b)

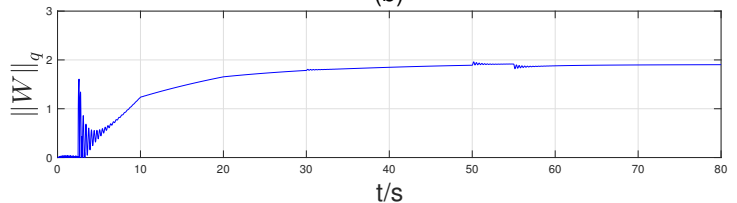

(c)

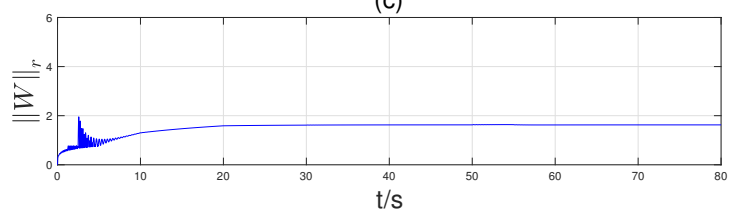

Fig. 20 Time histories for Frobenius norm of weight matrices to attitude control

varying working capability. It has been proven in terms of Lyapunov theorem that the proposed control scheme achieves the compound helicopter multimodal tracking objective with an asymptotically stable performance. Numerical simulation finally has verified the efficacy of the proposed approach.

\section{Declarations}

\section{Funding}

This work is supported by the National Natural Science Foundation of China (NOs. 61803200,61973158).

\section{Compliance with ethical standards}

\section{Conflict of interest}

The authors declare that they have no conflict of interest.

\section{Availability of data and material}

\section{Code availability}

\section{References}

1. Sugawara, H., Y. Tanabe, M. Kameda.: Effect of Lift-Share Ratio on Aerodynamic Performance of Winged Compound Helicopter. Journal of Aircraft. 1, 1-13(2021). https://doi.org/10.2514/1.C036163

2. Pölzlbauer P., Kümmel A., Desvigne D., Breitsamter C.: Numerical Investigation of an Optimized Rotor Head Fairing for the RACER Compound Helicopter in Cruise Flight. Aerospace. 8(3), 66(2021). https://doi.org/10.3390/aerospace8030066
3. K Yang, D. Han, Q. Shi.: Study on the lift and propulsive force shares to improve the flight performance of a compound helicopter. Chinese Journal of Aeronautics. 1(2021). https://doi.org/10.1016/j.cja.2021.02.010

4. Yuan, Y., Thomson, D., Chen, R.: Variable rotor speed strategy for coaxial compound helicopters with lift-offset rotors. The Aeronautical Journal. 124(1271), 96-120(2020). https://doi.org/10.1017/aer.2019.113

5. Thiemeier, J., Öhrle, C., Frey, F. et al.: Aerodynamics and flight mechanics analysis of Airbus Helicopters' compound helicopter RACER in hover under crosswind conditions. CEAS Aeronaut J. 11, 49-66 (2020). https://doi.org/10.1007/s13272-019-00392-3

6. Wilke G A.: Variable-Fidelity Methodology for the Aerodynamic Optimization of Helicopter Rotors. AIAA Journal. 57(8), 114(2019). https://doi.org/10.2514/1.J056486

7. Yuepeng, BU, Wenping, et al.: Aerodynamic/aeroacoustic variablefidelity optimization of helicopter rotor based on hierarchical Krig ing model. Chinese Journal of Aeronautics. 33(2), 476-492(2020). https://doi.org/10.1016/j.cja.2019.09.019

8. Hrle C, F Frey, Thiemeier J, et al.: Compound Helicopter $\mathrm{X} 3$ in High-Speed Flight: Correlation of Simulation and Flight Test. Journal of the American Helicopter Society. (2021). https://doi.org/10.4050/JAHS.66.012011

9. Yuan Y., Thomson D, R Chan: Variable rotor speed strategy for coaxial compound helicopters with lift-offset rotors. The Aeronautical Journal. 124(1271), 96-120(2020). https://doi.org/10.1007/10.1017/aer.2019.113

10. Linton D, Widjaja R, Thornber B.: Actuator Surface Model with Computational-Fluid-Dynamics-Convected Wake Model for Rotorcraft Application. AIAA Journal. 1,1-15(2021). https://doi.org/10.2514/1.J059952

11. Park J S.: Vibration Reduction Simulations for Rotor and Airframe of a Lift-offset Compound Helicopter Using Two Active Vibration Control Technique. Aerospace Science and Technology. 106(2020). https://doi.org/10.1016/j.ast.2020.106181

12. V.M.Kuvshinov.: Design of integral control algorithms for fly-by-wire control system of advanced high-speed helicopter. TsAGI science journal. 47(1), 95-109(2016). https://doi.org/10.1615/TsAGISciJ.2016017070

13. Seo J, Lee S, Lee J, et al.: Nonaffine Helicopter Control Design and Implementation Based on a Robust Explicit Nonlinear Model Predictive Control. IEEE Trans. on Control Syst. Technol. PP(99), 1-8(2021). https://doi.org/10.1109/TCST.2021.3069106

14. Bauersfeld L, Spannagl L , Ducard G, et al.: MPC Flight Control for a Tilt-rotor VTOL Aircraft. IEEE Trans. on Aerospace and Electronic Systems. (2021). https://doi.org/10.1109/TAES.2021.3061819

15. Woo G, Glezer A, Yorish S, et al.: Pulsed Actuation Control of Flow Separation on a ROBIN Rotorcraft Fuselage. Aiaa Journal. 254(10), 1-16(2016). https://doi.org/10.2514/1.J054008

16. Brescianini D, D'Andrea R.: Tilt-Prioritized Quadrocopter Attitude Control. IEEE Trans. on Control Syst. Technol. 28(2), 112(2018). https://doi.org/10.1109/TCST.2018.2873224

17. Guerreiro B J, Silvestre C, Cunha R, et al.: LiDAR-based Control of Autonomous Rotorcraft for the Inspection of Pier-like Structures. IEEE Trans. on Control Syst. Technol. 26(4), 1430-1438(2018). https://doi.org/10.1109/TCST.2017.2705058

18. Chen, M., Yu, J.: Disturbance observer-based adaptive sliding mode control for near-space vehicles. Nonlinear Dyn. 82, 1671-1682 (2015). https://doi.org/10.1007/s11071-015-2268-x

19. Zong, Q., Wang, F., Tian, B. et al.: Robust adaptive dynamic surface control design for a flexible air-breathing hypersonic vehicle with input constraints and uncertainty. Nonlinear Dyn. 78, 289-315 (2014). https://doi.org/10.1007/s11071-014-1440-z

20. An, H., Wu, Q.: Switched-model-based compound control of hypersonic vehicles with input nonlinearities. Nonlinear Dyn. 98, 463-476 (2019). https://doi.org/10.1007/s11071-019-05204-1 
21. Sheng, Y., Bai, W., Xie, Y. Fractional-order $P I^{\lambda} D$ sliding mode control for hypersonic vehicles with neural network disturbance compensator. Nonlinear Dyn. 103, 849-863 (2021) https://doi.org/10.1007/s11071-020-06046-y

22. Yao, D., Dou, C., Yue, D. et al.: Adaptive neural network consensus tracking control for uncertain multi-agent systems with predefined accuracy. Nonlinear Dyn. 101, 2249-2262 (2020). https://doi.org/10.1007/s11071-020-05885-z

23. Guo X G, Xu W D, Wang $J$ L, et al.: Distributed Neuroadaptive Fault-tolerant Sliding-mode Control for 2-D Plane Vehicular Platoon Systems with Spacing Constraints and Unknown Direction Faults. Automatica. 129, 109675(2021). https://doi.org/10.1016/j.automatica.2021.109675

24. Zhang Q, Wang H.: A Novel Data-based Stochastic Distribution Control for Non-Gaussian Stochastic Systems. IEEE Trans. on Autom. Control. (2021). https://doi.org/10.1109/TAC.2021.3064991

25. Wang $M$, Wang $Z$, Dong $H$, et al.: A Novel Framework for Backstepping-Based Control of Discrete-Time StrictFeedback Nonlinear Systems With Multiplicative Noises. IEEE Trans. on Autom. Control. 66(4), 1484-1496(2020). https://doi.org/10.1109/TAC.2020.2995576

26. Funahashi K I.: On the approximate realization of continuous mappings by neural networks. Neural Networks. 2(3):183192(1989). https://doi.org/10.1016/0893-6080(89)90003-8

27. Hornik K, Stinchcombe M, White H.: Multilayer feedforward networks are universal approximators. Neural Networks. 2(5), 359366(1989). https://doi.org/10.1016/0893-6080(89)90020-8

28. Hunt K J, Sbarbaro D, Bikowski R, et al.: Neural networks for control systems-A survey. Automatica. 28(6), 1083-1112(1992). https://doi.org/10.1016/0005-1098(92)90053-I

29. Rysdyk R, Calise A J.: Robust nonlinear adaptive flight control for consistent handling qualities.IEEE Trans. on Control Syst. Technol. 13(6), 896-910(2005). https://doi.org/10.1109/TCST.2005.854345

30. Zhang C, Yang J, Wu W.: Binary Higher Order Neural Networks for Realizing Boolean Functions. IEEE Trans. on Neural Networks. 22(5), 701-713(2011). https://doi.org/10.1109/tnn.2011.2114367

31. Kang Q, Fan Q, Zurada J M.: Deterministic Convergence Analysis via Smoothing Group Lasso Regularization and Adaptive Momentum for Sigma-Pi-Sigma Neural Network. Information Sciences. 553, 66-82(2020) https://doi.org/10.1016/j.ins.2020.12.014

32. Yilmaz, O., Bas, E. Egrioglu, E.: The Training of Pi-Sigma Artificial Neural Networks with Differential Evolution Algorithm for Forecasting. Comput. Econ. 2021. https://doi.org/10.1007/s10614020-10086-2

33. Panda N, Majhi S K.: Improved spotted hyena optimizer with space transformational search for training pi-sigma higher order neural network. Computational Intelligence. 36(1), 320-350(2020). https://doi.org/10.1111/coin.12272

34. Bas E, Grosan C, Egrioglu E, et al.: High order fuzzy time series method based on pi-sigma neural network. Engineering Applications of Artificial Intelligence. 72(JUN), 350-356(2018). https://doi.org/10.1016/j.engappai.2018.04.017

35. Akdeniz E, Egrioglu E, Bas E, et al.: An ARMA Type Pi-Sigma Artificial Neural Network for Nonlinear Time Series Forecasting. Journal of Artificial Intelligence and Soft Computing Research. 8(2), 121-132(2018). https://doi.org/10.1515/jaiscr-2018-0009

36. Eren B, Crina G, Erol E, et al.: High order fuzzy time series method based on pi-sigma neural network. Engineering Applications of Artificial Intelligence. 72, 350-356(2018) https://doi.org/10.1016/j.engappai.2018.04.017

37. Xu R, Zhou M.: A self-adaption compensation control for hysteresis nonlinearity in piezo-actuated stages based on Pi-sigma fuzzy neural network. Smart Mater. Struct. 27(4), 045002(2018). https://doi.org/10.1088/1361-665X/aaae28

38. Shen W, Shen $\mathrm{C}, \mathrm{Su} X$, et al.: Finite-time command filtered control combined with pi-sigma fuzzy neural network for hydraulic control system. Asian Journal of Control. 1-20(2020) https://doi.org/10.1002/asjc. 2400

39. Fengying $\mathrm{Z}$, Longwu L, et al.: Hybrid multi-objective control allocation strategy for compound high-speed rotorcraft. ISA Trans. 98, 207-226(2020). https://doi.org/10.1016/j.isatra.2019.08.039 